\title{
Bayesian estimation of 3-component mixture of Gumbel type-II distributions under non-informative and informative priors
}

\author{
Tabasam Sultana ${ }^{1 *}$, Muhammad Aslam ${ }^{2}$ and Mariya Raftab ${ }^{1}$ \\ ${ }^{1}$ Department of Statistics, Faculty of Natural Sciences, Quaid-i-Azam University, Islamabad, Pakistan. \\ ${ }^{2}$ Department of Basic Sciences, Faculty of Natural Sciences, Ripha International University, Islamabad, Pakistan.
}

Revised: 26 December 2016; Accepted: 16 February 2017

\begin{abstract}
This paper deals with 3-component mixture of the Gumbel type-II distributions when the scale parameter is known under Bayesian view point. The type-I right censored sampling scheme is considered due to its extensive use in reliability theory and survival analysis, taking different non-informative and informative priors. Bayes estimates of the parameters of the mixture model along with their posterior risks are derived under different loss functions. In case where no or little prior information is available, elicitation of hyperparameters is given. In order to numerically study the execution of the Bayes estimators under different loss functions, their statistical properties have been simulated for different sample sizes and test termination times. The comparisons among the estimators have been made in terms of the corresponding posterior risks. A real life data example is also given to illustrate the study.
\end{abstract}

Keywords: Bayes estimators, censoring, loss functions, mixture models, posterior risks.

\section{INTRODUCTION}

The Gumbel distribution was introduced by the German mathematician Emil Gumbel in 1958, and is useful in predicting the chance of meteorological phenomena, such as annual flood flows, earthquakes and other natural disasters. It has also been used in describing the life expectancy of components. In hydrology, the Gumbel distribution is used to analyse the variables such as monthly, quarterly and annual maximum values of daily rainfall, wind speed and river discharge volumes. The Gumbel distribution is a special case of the generalised extreme value distribution (also identified as the FisherTippett distribution), and the distribution is also known as the log-Weibull distribution and the double exponential distribution (also considered as the Laplace distribution).
Many authors have discussed the Gumbel type-II distribution. For example, Kotz and Nadarajah (2000) investigated some properties of the Gumbel distribution. Feroze and Aslam (2012) applied the Bayesian estimation scheme for Gumbel type-II distribution under doubly censored samples by considering various loss functions. Furthermore, Salinas et al. (2012) proposed goodness-of-fit tests for the Gumbel distribution with type-II right censored data. Abbas et al. (2013) obtained the Bayes estimators for the parameters of the Gumbel type-II distribution under different loss functions and compared these estimates with similar performance by the maximum likelihood method. Reyad and Ahmed (2015) proposed the E-Bayesian analysis of the unknown shape parameter of the Gumbel type-II distribution based on type-II censored samples. These estimators are obtained under different symmetric and asymmetric loss functions.

Mixture models play an important role in many applicable fields such as medicine, psychology, cluster analysis, life testing and reliability analysis. A finite mixture of some suitable probability distributions is recommended to study a population that is supposed to comprise a number of subpopulations mixing in unknown proportion. However, several researchers are interested in different parameters of mixture distributions. Chen et al. (1989) considered the Bayes estimation for mixtures of two Weibull distributions under type-I censoring. Saleem et al. (2010) presented the Bayesian analysis of the 2-component mixture of power function distributions using complete and censored data. Kazmi et al. (2012) developed the Bayesian analysis of 2-component mixture of the Maxwell distributions, while Feroze 
and Aslam (2013) discussed the Bayesian estimation of 2-component mixture of Gumbel type-II distribution under informative priors using different loss functions. Noor and Aslam (2013) studied Bayesian inference of the inverse Weibull mixture model under type-I censoring, and Ali (2015) described mixture of inverse Rayleigh distribution under Bayesian framework.

One particular feature, often present in time-to-event data, is known as censoring. Which broadly speaking, occurs when some lifetimes are known to have occurred only within certain intervals. The remainders of the lifetimes are known exactly. There are various categories of censoring such as right censoring, left censoring and interval censoring, single or multiple censoring, and type-I and type-II censoring. Type-I and type-II censoring schemes are most familiar among them.

Motivated by the above mentioned applications of mixture models, the authors have planned to conduct Bayesian analysis of a 3-component mixture of the Gumbel type-II distributions with unknown mixing proportions. The parameters of component distributions are assumed to be unknown. Three different priors and three different loss functions are used for the Bayesian analysis. In addition, an ordinary type-I right censored sampling scheme is also assumed.

\section{METHODOLOGY}

\section{3-component mixture of Gumbel type-II distributions}

The probability density function (PDF) and the cumulative distribution function (CDF) of the Gumbel type-II distribution for a random variable $x$ are given by:

$$
\begin{aligned}
& f_{m}\left(x ; \alpha_{m}, \beta_{m}\right)=\alpha_{m} \beta_{m} x^{-\left(\alpha_{m}+1\right)} \exp \left(-\beta_{m} x^{-\alpha_{m}}\right), x \geq 0, \alpha_{m}> \\
& 0, \beta_{m}>0, m=1,2,3 \\
& F_{m}(x)=1-\exp \left(-\beta_{m} x^{-\alpha_{m}}\right), m=1,2,3
\end{aligned}
$$

Where $\alpha_{m}$ and $\beta_{m}$ are the parameters of Gumble type-II distribution.

A finite 3-component mixture model with the unknown mixing proportions $p_{1}$ and $p_{2}$ is defined as when the scale parameter $\alpha_{m}=1$

$$
\begin{aligned}
& f(x)=p_{1} f_{1}(x)+p_{2} f_{2}(x)+\left(1-p_{1}-p_{2}\right) f_{3}(x) \\
& p_{1}, p_{2} \geq 0, p_{1}+p_{2} \leq 1
\end{aligned}
$$

$$
\begin{aligned}
& f\left(x ; \beta_{1}, \beta_{2}, \beta_{3} p_{1}, p_{2}\right)=p_{1} \beta_{1} x^{-2} \exp \left(-\beta_{1} x^{-1}\right)+p_{2} \beta_{2} x^{-2} \\
& \exp \left(-\beta_{2} x^{-1}\right)+\left(1-p_{1}-p_{2}\right) \beta_{3} x^{-2} \exp \left(-\beta_{3} x^{-1}\right)
\end{aligned}
$$

While the CDF of 3-component mixture of the Gumbel type-II distributions is given by:

$$
\begin{gathered}
F(x)=p_{1} F_{1}(x)+p_{2} F_{2}(x)+\left(1-p_{1}-p_{2}\right) F_{3}(x) \\
F(x)=1-p_{1} \exp \left(-\beta_{1} x^{-1}\right)-p_{2} \exp \left(-\beta_{2} x^{-1}\right)- \\
\left(1-p_{1}-p_{2}\right) \exp \left(-\beta_{3} x^{-1}\right)
\end{gathered}
$$

\section{The likelihood function}

Suppose ' $\mathrm{n}$ ' units from the 3-component mixture of Gumbel type-II distributions are used in a life testing experiment with fixed test termination time ' $t$ '. Let ' $r$ ' units out of ' $n$ ' units failed until fixed test termination time ' $\mathrm{t}$ ' and the remaining (n-r) units are still working. According to Mendenhall and Hader (1958), there are many practical situations in which the failed objects can be pointed out easily as a subset of subpopulation-I, subpopulation-II or subpopulation-III. Out of ' $\mathrm{r}$ ' units, suppose $r_{1}, r_{2}$ and $r_{3}$ units belong to subpopulation-I, subpopulation-II or subpopulation-III, respectively and such that $r=r_{1}+r_{2}+r_{3}$. Now we define $x_{l k}, 0<x_{l k}<t$ be the failure time of $k^{\text {th }}$ unit belonging to the $l^{\text {th }}$ subpopulation, where $l=1,2,3$ and $k=1,2, \ldots, r_{l}$. For a 3-component mixture model, the likelihood function can be written as

$$
\begin{aligned}
L\langle\psi \mid x\rangle \propto & \left\{\prod_{k=1}^{r_{1}} p_{1} f_{1}\left(x_{1 k}\right)\right\}\left\{\prod_{k=1}^{r_{2}} p_{2} f_{2}\left(x_{2 k}\right)\right\} \\
& \left\{\prod_{k=1}^{r_{3}}\left(1-p_{1}-p_{2}\right) f_{3}\left(x_{3 k}\right)\right\}[1-F(t)]^{n-r}
\end{aligned}
$$

After simplification, the likelihood function of 3-component mixture of Gumbel type-II distributions is given by:

$$
\begin{aligned}
& L\langle\psi \mid x\rangle \propto \beta_{1}^{r_{1}} \beta_{2}^{r_{2}} \beta_{3}^{r_{3}} \sum_{i=0}^{n-r} \sum_{j=0}^{i}\left(\begin{array}{l}
n-r \\
i
\end{array}\right)\left(\begin{array}{l}
i \\
j
\end{array}\right) \exp \left\{-\beta_{1}\left((n-r-i) t^{-1}+\sum_{k=1}^{r_{1}} x_{1 k}^{-1}\right)\right\} \\
& \exp \left\{-\beta_{2}\left((i-j) t^{-1}+\sum_{k=1}^{r_{2}} x_{2 k}^{-1}\right)\right\} \exp \left\{-\beta_{3}\left((j) t^{-1}+\sum_{k=1}^{r_{3}} x_{3 k}^{-1}\right)\right\} \\
& p_{1}^{n-r-i+r_{1}} p_{2}^{i-j+r_{2}}\left(1-p_{1}-p_{2}\right)^{j+r_{3}}
\end{aligned}
$$

Where $X=\left(x_{11}, x_{12}, \ldots, x_{1 r_{1}}, x_{21}, x_{22}, \ldots, x_{2 r_{2}}, x_{31}, x_{32}, \ldots, x_{3 r_{3}}\right)$ are the observed failure times for the uncensored observations and $\psi=\left(\beta_{1}, \beta_{2}, \beta_{3}, p_{1}, p_{2}\right)$. 
Posterior distribution using the non-informative and the informative priors (IP)

In this section, posterior distributions of parameters given data, say $x$, are derived using the non-informative (uniform and Jeffreys) and the informative (gamma) priors.

\section{Posterior distribution using uniform prior (UP)}

When the elicitation of hyperparameters is difficult or little prior information is given, usually the noninformative prior is assumed to be the UP. UPs over the intervals $(0, \infty)$ and $(0,1)$ are taken for the parameters $\beta_{1}, \beta_{2}$ and $\beta_{3}$ of Gumble type-II distribution and for the mixing proportions $\left(p_{1}, p_{2}\right)$, respectively. With these settings, joint prior distribution of parameters $\left(\beta_{1}, \beta_{2}, \beta_{3}\right.$, $\left.p_{1}, p_{2}\right)$, as defined by Saleem et al. (2010), is given by:

$$
\pi_{1}(\psi) \propto 1 ; \beta_{1}, \beta_{2}, \beta_{3}>0, p_{1}, p_{2} \geq 0, p_{1}+p_{2} \leq 1
$$

The joint posterior distribution of parameters $\beta_{1}, \beta_{2}, \beta_{3}$, $p_{1}$ and $p_{2}$ given data $x$ assuming the UP is:

$$
g_{1}\langle\psi \mid x\rangle=\frac{L\langle\psi \mid x\rangle \pi_{1}(\psi)}{\int_{\psi} L\langle\psi \mid x\rangle \pi_{1}(\psi) d \psi}
$$

By using equations (8) and (9) in equation (10), we can obtain the following:

$$
\begin{aligned}
& g_{1}\langle\psi \mid x\rangle=Q_{1}^{-1} \sum_{i=0}^{n-r} \sum_{j=0}^{i}\left(\begin{array}{l}
n-r \\
i
\end{array}\right)\left(\begin{array}{l}
i \\
j
\end{array}\right) \beta_{1}^{A_{11}-1} \beta_{2}^{A_{21}-1} \beta_{3}^{A_{31}-1} \\
& \exp \left(-\beta_{1} B_{11}\right) \exp \left(-\beta_{2} B_{21}\right) \exp \left(-\beta_{3} B_{31}\right) \\
& P_{1}^{A_{01}-1} p_{2}^{B_{01}-1}\left(1-p_{1}-p_{2}\right)^{C_{01}-1} \\
& A_{11}=r_{1}+1, A_{21}=r_{2}+1, A_{31}=r_{3}+1, B_{11}=\sum_{k=1}^{r_{1}} x_{1 k}^{-1}+(n-r-i) t^{-1}, \\
& B_{21}=\sum_{k=1}^{r_{2}} x_{2 k}^{-1}+(i-j) t^{-1}, B_{31}=\sum_{k=1}^{r_{3}} x_{3 k}^{-1}+j t^{-1}, \\
& A_{01}=n-r-i+r_{1}+1, B_{01}=i-j+r_{2}+1, C_{01}=j+r_{3}+1, \\
& Q_{1}=\sum_{i=0}^{n-r} \sum_{j=0}^{i}\left(\begin{array}{c}
n-r \\
i
\end{array}\right)\left(\begin{array}{c}
i \\
j
\end{array}\right) B\left(A_{01}, C_{01}\right) B\left(B_{01}, A_{01}+C_{01}\right) \\
& B_{11}^{A_{11}} \frac{\Gamma\left(A_{21}\right)}{B_{21}^{A_{21}}} \frac{\Gamma\left(A_{31}\right)}{B_{31}^{A_{31}}}
\end{aligned}
$$

\section{Posterior distribution using the Jeffreys prior (JP)}

According to Jeffreys (1946; 1961) and Berger (1985), the JP is defined as, where $p\left(\beta_{m}\right) \propto \sqrt{\left|I\left(\beta_{m}\right)\right|}$, where $I\left(\beta_{m}\right)=-E\left[\frac{\partial^{2} f\left(x, \beta_{m}\right)}{\partial \beta_{m}^{2}}\right]$ is the Fisher's information matrix. The prior distributions of the mixing proportions $p_{1}$ and $p_{2}$ are again taken to be uniform over the interval $(0,1)$. The joint prior distribution of parameters $\beta_{1}, \beta_{2}, \beta_{3}, p_{1}$ and $p_{2}$ is:

$$
\pi_{2}(\psi) \propto \frac{1}{\beta_{1} \beta_{2} \beta_{3}}, \beta_{1}, \beta_{2}, \beta_{3} \geq 0, p_{1}, p_{2} \geq 0, p_{1}+p_{2} \leq 1,
$$

The joint posterior distribution of parameters $\beta_{1}, \beta_{2}, \beta_{3}, p_{1}$ and $p_{2}$ given data $x$ assuming the JP is:

$$
g_{2}\langle\psi \mid x\rangle=\frac{L\langle\psi \mid x\rangle \pi_{2}(\psi)}{\int_{\psi} L\langle\psi \mid x\rangle \pi_{2}(\psi) d \psi}
$$

By using equations (8) and (12) in equation (13), we can obtain the following:

$$
\begin{aligned}
& g_{2}\langle\psi \mid x\rangle=Q_{2}^{-1} \sum_{i=0}^{n-r} \sum_{j=0}^{i}\left(\begin{array}{l}
n-r \\
i
\end{array}\right)\left(\begin{array}{l}
i \\
j
\end{array}\right) \beta_{1}^{A_{12}-1} \beta_{2}^{A_{22}-1} \beta_{3}^{A_{32}-1} \\
& \exp \left(-\beta_{1} B_{12}\right) \exp \left(-\beta_{2} B_{22}\right) \exp \left(-\beta_{3} B_{32}\right) \\
& p_{1}^{A_{02}-1} p_{2}^{B_{02}-1}\left(1-p_{1}-p_{2}\right)^{C_{02}-1}
\end{aligned}
$$

where
$A_{12}=r_{1}, A_{22}=r_{2}, A_{32}=r_{3}, B_{12}=\sum_{k=1}^{r_{1}} x_{1 k}^{-1}+(n-r-i) t^{-1}$,

$B_{22}=\sum_{k=1}^{r_{2}} x_{2 k}^{-1}+(i-j) t^{-1}$

$B_{32}=\sum_{k=1}^{r_{3}} x_{3 k}^{-1}+j t^{-1}, A_{02}=n-r-i+r_{1}+1, B_{02}=i-j+r_{2}+1$,

$C_{02}=j+r_{3}+1$,

$Q_{2}=\sum_{i=0}^{n-r} \sum_{j=0}^{i}\left(\begin{array}{l}n-r \\ i\end{array}\right)\left(\begin{array}{l}i \\ i\end{array}\right) B\left(A_{02}, C_{02}\right) B\left(B_{02}, A_{02}+C_{02}\right) \frac{\Gamma\left(A_{12}\right)}{B_{12}^{A_{12}}} \frac{\Gamma\left(A_{22}\right)}{B_{22}^{A_{22}}} \frac{\Gamma\left(A_{32}\right)}{B_{32}^{A_{32}}}$

\section{Posterior distribution using the gamma prior (GP)}

As an informative prior, we take the gamma prior for the component parameters $\beta_{1}, \beta_{2}, \beta_{3}$ and bivariate beta prior for proportion parameters $p_{1}, p_{2}$. Symbolically, it can be written as: $\beta_{1} \sim \operatorname{Gamma}\left(a_{1}, b_{1}\right), \beta_{2} \sim \operatorname{Gamma}\left(a_{2}, b_{2}\right), \beta_{3} \sim \operatorname{Gamma}\left(a_{3}, b_{3}\right)$ and $p_{1}, p_{2} \sim$ bivariate beta $(a, b, c)$. Again assuming 
independence of all parameters, the joint prior distribution of $\left(\beta_{1}, \beta_{2}, \beta_{3}, p_{1}, p_{2}\right)$ is given by:

$\pi_{3}(\phi) \propto \beta_{1}^{a_{1}-1} \exp \left(-b_{1} \beta_{1}\right) \beta_{2}^{a_{2}-1} \exp \left(-b_{2} \beta_{2}\right) \beta_{3}^{a_{3}-1}$

$\exp \left(-b_{3} \beta_{3}\right) p_{1}^{a-1} p_{2}^{b-1}\left(1-p_{1}-p_{2}\right)^{c-1}$

The joint posterior distribution of parameters $\beta_{1}, \beta_{2}, \beta_{3}, p_{1}$ and $p_{2}$ given data $x$

$g_{3}\langle\psi \mid x\rangle=\frac{L\langle\psi \mid x\rangle \pi_{3}(\psi)}{\int_{\psi} L\langle\psi \mid x\rangle \pi_{3}(\psi) d \psi}$

By using equations (8) and (15) in equation (16), we can obtain the following:

$g_{3}\langle\psi \mid x\rangle=Q_{3}^{-1} \sum_{i=0}^{n-r} \sum_{j=0}^{i}\left(\begin{array}{l}n-r \\ i\end{array}\right)\left(\begin{array}{l}i \\ j\end{array}\right) \beta_{1}^{A_{13}-1} \beta_{2}^{A_{23}-1} \beta_{3}^{A_{33}-1}$

$\exp \left(-\beta_{1} B_{13}\right) \exp \left(-\beta_{2} B_{23}\right) \exp \left(-\beta_{3} B_{33}\right)$

$p_{1}^{A_{03}-1} p_{2}^{B_{03}-1}\left(1-p_{1}-p_{2}\right)^{C_{03}-1}$

where

$$
\begin{aligned}
& A_{13}=r_{1}+a_{1}, A_{23}=r_{2}+a_{2}, A_{33}=r_{3}+a_{3}, \\
& B_{13}=\sum_{k=1}^{r_{1}} x_{1 k}^{-1}+(n-r-i) t^{-1}+b_{1}, B_{23}=\sum_{k=1}^{r_{2}} x_{2 k}^{-1}+(i-j) t^{-1}+b_{2}, \\
& B_{33}=\sum_{k=1}^{r_{3}} x_{3 k}^{-1}+j t^{-1}+b_{3}, A_{03}=n-r-i+r_{1}+a, B_{03}=i-j+r_{2}+b, \\
& C_{03}=j+r_{3}+c, \\
& Q_{3}=\sum_{i=0}^{n-r} \sum_{j=0}^{i}\left(\begin{array}{l}
n-r \\
i
\end{array}\right)\left(\begin{array}{l}
i \\
j
\end{array}\right) B\left(A_{03}, C_{03}\right) B\left(B_{03}, A_{03}+C_{03}\right) \frac{\Gamma\left(A_{13}\right)}{B_{13}^{A_{13}}} \frac{\Gamma\left(A_{23}\right)}{B_{23}^{A_{23}}} \frac{\Gamma\left(A_{33}\right)}{B_{33}^{A_{33}}}
\end{aligned}
$$

Bayes estimators and posterior risks using the UP, JP and IP under different loss functions

If $L(\beta, d)$ is a loss function, then the expected value of the loss function for a given decision with respect to the posterior distribution is posterior risk function, and if $\hat{d}$ is a Bayes estimator, then $\rho(\hat{d})$ is called posterior risk and is given by $\rho(\hat{d})=E_{\beta \mid x}\{L(\beta, d)\}$.

Bayes estimators and posterior risks using the UP, JP and IP under SELF

The squared error loss function (SELF) has been suggested by Legendre (1805), and is defined as: $L(\beta, d)=(\beta-d)^{2}$. The Bayes estimator and posterior risk under SELF are: $\hat{d}=E_{\beta \mid x}(\beta)$ and $\rho(\hat{d})=E_{\beta \mid x}\left(\beta^{2}\right)-\left\{E_{\beta \mid x}(\beta)\right\}^{2}$, respectively.
So, the Bayes estimators and posterior risks using the UP, JP and IP for parameters $\beta_{1}, \beta_{2}, \beta_{3}, p_{1}$ and $p_{2}$ under SELF obtained with their respective marginal posterior distributions are given below:

$\hat{\beta}_{1 \tau}=Q_{\tau}^{-1} \sum_{i=0}^{n-r} \sum_{j=0}^{i}\left(\begin{array}{l}n-r \\ i\end{array}\right)\left(\begin{array}{l}i \\ j\end{array}\right) \frac{\Gamma\left(A_{1 \tau}+1\right)}{B_{1 \tau}^{A_{1}+1}} \frac{\Gamma\left(A_{2 \tau}\right)}{B_{2 \tau}^{A_{2 \tau}}} \frac{\Gamma\left(A_{3 \tau}\right)}{B_{3 \tau}^{A_{\tau}}} B\left(A_{0 \tau}, C_{0 \tau}\right) B\left(B_{0 \tau}, A_{0 \tau}+C_{0 \tau}\right)$

$\hat{\beta}_{2 \tau}=Q_{\tau}^{-1} \sum_{i=0}^{n-r} \sum_{j=0}^{i}\left(\begin{array}{l}n-r \\ i\end{array}\right)\left(\begin{array}{l}i \\ j\end{array}\right) \frac{\Gamma\left(A_{1 \tau}\right)}{B_{1 \tau}^{A_{1 \tau}}} \frac{\Gamma\left(A_{2 \tau}+1\right)}{B_{2 \tau}^{A_{\tau}+1}} \frac{\Gamma\left(A_{3 \tau}\right)}{B_{3 \tau}^{A_{\tau} \tau}} B\left(A_{0 \tau}, C_{0 \tau}\right) B\left(B_{0 \tau}, A_{0 \tau}+C_{0 \tau}\right)$

$\hat{\beta}_{3 \tau}=Q_{\tau}^{-1} \sum_{i=0}^{n-r} \sum_{j=0}^{i}\left(\begin{array}{l}n-r \\ i\end{array}\right)\left(\begin{array}{l}i \\ j\end{array}\right) \frac{\Gamma\left(A_{1 \tau}\right)}{B_{1 \tau}^{A_{1 \tau}}} \frac{\Gamma\left(A_{2 \tau}\right)}{B_{2 \tau}^{A_{2 \tau}}} \frac{\Gamma\left(A_{3 \tau}+1\right)}{B_{3 \tau}^{A_{\tau}+1}} B\left(A_{0 \tau}, C_{0 \tau}\right) B\left(B_{0 \tau}, A_{0 \tau}+C_{0 \tau}\right)$

$\hat{p}_{1 \tau}=Q_{\tau}^{-1} \sum_{i=0}^{n-r} \sum_{j=0}^{i}\left(\begin{array}{l}n-r \\ i\end{array}\right)\left(\begin{array}{l}i \\ j\end{array}\right) \frac{\Gamma\left(A_{1 \tau}\right)}{B_{1 \tau}^{A_{1 \tau}}} \frac{\Gamma\left(A_{2 \tau}\right)}{B_{2 \tau}^{A_{2 \tau}}} \frac{\Gamma\left(A_{3 \tau}\right)}{B_{3 \tau}^{A_{3 \tau}}} B\left(B_{0 \tau}, C_{0 \tau}\right) B\left(A_{0 \tau}+1, B_{0 \tau}+C_{0 \tau}\right)$

$\hat{p}_{2 \tau}=Q_{\tau}^{-1} \sum_{i=0}^{n-r} \sum_{j=0}^{i}\left(\begin{array}{l}n-r \\ i\end{array}\right)\left(\begin{array}{l}i \\ j\end{array}\right) \frac{\Gamma\left(A_{1 \tau}\right)}{B_{1 \tau}^{A_{1 \tau}}} \frac{\Gamma\left(A_{2 \tau}\right)}{\left.B_{2 \tau}\right)} \frac{\Gamma\left(A_{3 \tau}\right)}{B_{3 \tau}^{A_{3 \tau}}} B\left(A_{0 \tau}, C_{0 \tau}\right) B\left(B_{0 \tau}+1, A_{0 \tau}+C_{0 \tau}\right)$

$\rho\left(\hat{\beta}_{1 \tau}\right)=Q_{\tau}^{-1} \sum_{i=0}^{n-r} \sum_{j=0}^{i}\left(\begin{array}{l}n-r \\ i\end{array}\right)\left(\begin{array}{l}i \\ j\end{array}\right) \frac{\Gamma\left(A_{1 \tau}+2\right)}{B_{2 \tau}^{A_{1 \tau}+2}} \frac{\Gamma\left(A_{2 \tau}\right)}{B_{2 \tau}^{A_{\tau}}} \frac{\Gamma\left(A_{3 \tau}\right)}{B_{3 \tau}^{A_{3 \tau}}} B\left(A_{0 \tau}, C_{0 \tau}\right) B\left(B_{0 \tau}, A_{0 \tau}+C_{0 \tau}\right)-\left(\hat{\beta}_{1 \tau}\right)^{2}$

$\rho\left(\hat{\beta}_{2 \tau}\right)=Q_{\tau}^{-1} \sum_{i=0}^{n-r} \sum_{j=0}^{i}\left(\begin{array}{l}n-r \\ i\end{array}\right)\left(\begin{array}{l}i \\ j\end{array}\right) \frac{\Gamma\left(A_{1 \tau}\right)}{\left.B_{2 \tau}^{A_{\tau}}\right)} \frac{\Gamma\left(A_{2 \tau}+2\right)}{B_{2 \tau}^{A_{2 \tau}+2}} \frac{\Gamma\left(A_{3 \tau}\right)}{B_{3 \tau}^{A_{3 \tau}}} B\left(A_{0 \tau}, C_{0 \tau}\right) B\left(B_{0 \tau}, A_{0 \tau}+C_{0 \tau}\right)-\left(\hat{\beta}_{2 \tau}\right)^{2}$

$\left.\rho\left(\hat{\beta}_{3 \tau}\right)=Q_{\tau}^{-1} \sum_{i=0}^{n-r} \sum_{j=0}^{i}\left(\begin{array}{l}n-r \\ i\end{array}\right)\left(\begin{array}{l}i \\ j\end{array}\right) \frac{\Gamma\left(A_{1 \tau}\right)}{B_{2 \tau}^{A_{\tau}}}\right) \frac{\Gamma\left(A_{2 \tau}\right)}{B_{2 \tau}^{A_{2} \tau}} \frac{\Gamma\left(A_{3 \tau}+2\right)}{B_{3 \tau}^{\lambda_{3}+2}} B\left(A_{0 \tau}, C_{0 \tau}\right) B\left(B_{0 \tau}, A_{0 \tau}+C_{0 \tau}\right)-\left(\hat{\beta}_{3 \tau}\right)^{2}$

$\rho\left(\hat{p}_{1 \tau}\right)=Q_{\tau}^{-1} \sum_{i=0}^{n-r} \sum_{j=0}^{i}\left(\begin{array}{l}n-r \\ i\end{array}\right)\left(\begin{array}{l}i \\ j\end{array}\right) \frac{\Gamma\left(A_{1 \tau}\right)}{B_{2 \tau}^{A_{\tau}}} \frac{\Gamma\left(A_{2 \tau}\right.}{B_{2 \tau}^{A_{\tau} \tau}} \frac{\Gamma\left(A_{3 \tau}\right)}{B_{3 \tau}^{A_{\tau}}} B\left(B_{0 \tau}, C_{0 \tau}\right) B\left(A_{0 \tau}+2, B_{0 \tau}+C_{0 \tau}\right)-\left(\hat{p}_{1 \tau}\right)^{2}$

$\left.\rho\left(\hat{p}_{2 \tau}\right)=Q_{\tau}^{-1} \sum_{i=0}^{n-r} \sum_{j=0}^{i}\left(\begin{array}{l}n-r \\ i\end{array}\right)\left(\begin{array}{l}i \\ j\end{array}\right) \frac{\Gamma\left(A_{1 \tau}\right)}{B_{2 \tau}^{A_{\tau}}} \frac{\Gamma\left(A_{2 \tau}\right)}{B_{2 \tau}^{4}}\right) \frac{\Gamma\left(A_{3 \tau}\right)}{B_{3 \tau}^{A_{3}}} B\left(A_{0 \tau}, C_{0 \tau}\right) B\left(B_{0 \tau}+2, A_{0 \tau}+C_{0 \tau}\right)-\left(\hat{p}_{2 \tau}\right)^{2}$

Where $\tau=1$ for the uniform prior, $\tau=2$ for the Jeffreys prior and $\tau=3$ for the gamma prior. 
Bayes estimators and posterior risks using the UP, JP and IP under precautionary loss function (PLF)

Norstrøm (1996) discussed an asymmetric precautionary loss function (PLF) and also introduced a special case of general class of PLFs, which is defined as $L(\beta, d)=\frac{(\beta-d)^{2}}{d}$. The Bayes estimator and posterior risk are:

$\hat{d}=\left\{E_{\beta \mid x}\left(\beta^{2}\right)\right\}^{\frac{1}{2}} \& \rho(\hat{d})=2\left\{E_{\beta \mid x}\left(\beta^{2}\right)\right\}^{\frac{1}{2}}-2 E_{\beta \mid x}(\beta)$, respectively. The respective marginal posterior distribution yields the Bayes estimators and posterior risk using the UP, JP and IP for parameters $\beta_{1}, \beta_{2}, \beta_{3}, p_{1}$ and $p_{2}$ under PLF as:

$\hat{\beta}_{1 \tau}=\left\{Q_{\tau}^{-1} \sum_{i=0}^{n-r} \sum_{j=0}^{i}\left(\begin{array}{l}n-r \\ i\end{array}\right)\left(\begin{array}{l}i \\ j\end{array}\right) \frac{\Gamma\left(A_{1 \tau}+2\right)}{B_{1 \tau}^{A_{\tau}+2}} \frac{\Gamma\left(A_{2 \tau}\right)}{\left.B_{2 \tau}^{A_{\tau}}\right)} \frac{\Gamma\left(A_{3 \tau}\right)}{B_{3 \tau}^{A_{3 \tau}}} B\left(A_{0 \tau}, C_{0 \tau}\right) B\left(B_{0 \tau}, A_{0 \tau}+C_{0 \tau}\right)\right\}^{\frac{1}{2}}$

$\left.\hat{\beta}_{2 \tau}=\left\{Q_{\tau}^{-1} \sum_{i=0}^{n-r} \sum_{j=0}^{i}\left(\begin{array}{l}n-r \\ i\end{array}\right)\left(\begin{array}{l}i \\ j\end{array}\right) \frac{\Gamma\left(A_{1 \tau}\right)}{B_{1 \tau}^{A_{\tau}}}\right) \frac{\Gamma\left(A_{2 \tau}+2\right)}{B_{2 \tau}^{A_{2 \tau}+2}} \frac{\Gamma\left(A_{3 \tau}\right)}{B_{3 \tau}^{A_{3 \tau}}} B\left(A_{0 \tau}, C_{0 \tau}\right) B\left(B_{0 \tau}, A_{0 \tau}+C_{0 \tau}\right)\right\}$

$\hat{\beta}_{3 \tau}=\left\{Q_{\tau}^{-1} \sum_{i=0}^{n-r} \sum_{j=0}^{i}\left(\begin{array}{l}n-r \\ i\end{array}\right)\left(\begin{array}{l}i \\ j\end{array}\right) \frac{\Gamma\left(A_{1 \tau}\right)}{B_{1 \tau}^{A_{1 \tau}}} \frac{\Gamma\left(A_{2 \tau}\right)}{B_{2 \tau}^{A_{2 \tau}}} \frac{\Gamma\left(A_{3 \tau}+2\right)}{B_{3 \tau}^{A_{3 \tau}+2}} B\left(A_{0 \tau}, C_{0 \tau}\right) B\left(B_{0 \tau}, A_{0 \tau}+C_{0 \tau}\right)\right\}^{\frac{1}{2}}$

$\hat{p}_{1 \tau}=\left\{Q_{\tau}^{-1} \sum_{i=0}^{n-r} \sum_{j=0}^{i}\left(\begin{array}{l}n-r \\ i\end{array}\right)\left(\begin{array}{l}i \\ j\end{array}\right) \frac{\Gamma\left(A_{1 \tau}\right)}{B_{1 \tau}^{A_{\tau} \tau}} \frac{\Gamma\left(A_{2 \tau}\right)}{\left.B_{2 \tau}^{A_{2 \tau}}\right)} \frac{\Gamma\left(A_{3 \tau}\right)}{B_{3 \tau}^{A_{3 \tau}}} B\left(B_{0 \tau}, C_{0 \tau}\right) B\left(A_{0 \tau}+2, B_{0 \tau}+C_{0 \tau}\right)\right\}^{\frac{1}{2}}$

$\hat{p}_{2 \tau}=\left\{Q_{\tau}^{-1} \sum_{i=0}^{n-r} \sum_{j=0}^{i}\left(\begin{array}{l}n-r \\ i\end{array}\right)\left(\begin{array}{l}i \\ j\end{array}\right) \frac{\Gamma\left(A_{1 \tau}\right)}{B_{1 \tau}^{A_{1 \tau}}} \frac{\Gamma\left(A_{2 \tau}\right)}{B_{2 \tau}^{A_{2 \tau}}} \frac{\Gamma\left(A_{3 \tau}\right)}{B_{3 \tau}^{A_{3 \tau}}} B\left(A_{0 \tau}, C_{0 \tau}\right) B\left(B_{0 \tau}+2, A_{0 \tau}+C_{0 \tau}\right)\right\}^{\frac{1}{2}}$

$\rho\left(\hat{\beta}_{1}\right)=2\left\{Q_{\tau}^{-1} \sum_{i=0}^{n-r} \sum_{=0 j}^{i}\left(\begin{array}{l}n-r \\ i\end{array}\right)\left(\begin{array}{l}i \\ j\end{array}\right) \frac{\Gamma\left(A_{1 \tau}+2\right)}{B_{1 \tau}^{A_{1 \tau}+2}} \frac{\Gamma\left(A_{2 \tau}\right)}{B_{2 \tau}^{A_{2 \tau}}} \frac{\Gamma\left(A_{3 \tau}\right)}{B_{3 \tau}^{3_{3 \tau}}} B\left(A_{0 \tau}, C_{0 \tau}\right) B\left(B_{0 \tau}, A_{0 \tau}+C_{0 \tau}\right)\right\}$ $-2\left\{Q_{\tau}^{-1} \sum_{i=0}^{n-r} \sum_{j=0}^{i}\left(\begin{array}{l}n-r \\ i\end{array}\right)\left(\begin{array}{l}i \\ j\end{array}\right) \frac{\Gamma\left(A_{1 \tau}+1\right)}{B_{1 \tau}^{A_{\tau}+1}} \frac{\Gamma\left(A_{2 \tau}\right)}{B_{2 \tau}^{A_{\tau}}} \frac{\Gamma\left(A_{3 \tau}\right)}{B_{3 \tau}^{A_{\tau}}} B\left(A_{0 \tau}, C_{0 \tau}\right) B\left(B_{0 \tau}, A_{0 \tau}+C_{0 \tau}\right)\right\}$

$\left.\rho\left(\hat{\beta}_{2}\right)=2\left\{Q_{\tau}^{-1} \sum_{i=0}^{n-r} \sum_{=0 j}^{i}\left(\begin{array}{l}n-r \\ i\end{array}\right)\left(\begin{array}{l}i \\ j\end{array}\right) \frac{\Gamma\left(A_{1 \tau}\right)}{B_{1 \tau}^{A_{\tau}}}\right) \frac{\Gamma\left(A_{2 \tau}+2\right)}{B_{2 \tau}^{A_{\tau}+2}} \frac{\Gamma\left(A_{3 \tau}\right)}{B_{3 \tau}^{A_{3 \tau}}} B\left(A_{0 \tau}, C_{0 \tau}\right) B\left(B_{0 \tau}, A_{0 \tau}+C_{0 \tau}\right)\right\}^{\frac{1}{2}}$ $\left.-2\left\{Q_{\tau}^{-1} \sum_{i=0}^{n-r} \sum_{j=0}^{i}\left(\begin{array}{l}n-r \\ i\end{array}\right)\left(\begin{array}{l}i \\ j\end{array}\right) \frac{\Gamma\left(A_{1 \tau}\right)}{B_{1 \tau}^{A_{\tau}}}\right) \frac{\Gamma\left(A_{2 \tau}+1\right)}{B_{2 \tau}^{A_{\tau}+1}} \frac{\Gamma\left(A_{3 \tau}\right)}{B_{3 \tau}^{A_{\tau}}} B\left(A_{0 \tau}, C_{0 \tau}\right) B\left(B_{0 \tau}, A_{0 \tau}+C_{0 \tau}\right)\right\}$

$$
\begin{aligned}
& \rho\left(\hat{\beta}_{3}\right)=2\left\{Q_{\tau}^{-1} \sum_{i=0}^{n-r} \sum_{=0 j}^{i}\left(\begin{array}{l}
n-r \\
i
\end{array}\right)\left(\begin{array}{l}
i \\
j
\end{array}\right) \frac{\Gamma\left(A_{1 \tau}\right)}{\left.B_{1 \tau}^{A_{\tau}}\right)} \frac{\Gamma\left(A_{2 \tau}\right)}{B_{2 \tau}^{A_{2 \tau}}} \frac{\Gamma\left(A_{3 \tau}+2\right)}{B_{3 \tau}^{A_{\tau}+2}} B\left(A_{0 \tau}, C_{0 \tau}\right) B\left(B_{0 \tau}, A_{0 \tau}+C_{0 \tau}\right)\right\}^{\frac{1}{2}} \\
& -2\left\{Q_{\tau}^{-1} \sum_{i=0}^{n-r} \sum_{j=0}^{i}\left(\begin{array}{l}
n-r \\
i
\end{array}\right)\left(\begin{array}{l}
i \\
j
\end{array}\right) \frac{\Gamma\left(A_{1 \tau}\right)}{B_{1 \tau}^{A_{\tau}}} \frac{\Gamma\left(A_{2 \tau}\right)}{B_{2 \tau}^{A_{\tau}}} \frac{\Gamma\left(A_{3 \tau}+1\right)}{B_{3 \tau}^{A_{\tau}+1}} B\left(A_{0 \tau}, C_{0 \tau}\right) B\left(B_{0 \tau}, A_{0 \tau}+C_{0 \tau}\right)\right\}
\end{aligned}
$$

$$
\begin{aligned}
& \rho\left(\hat{p}_{1}\right)=2\left\{Q_{\tau}^{-1} \sum_{i=0}^{n-r} \sum_{=0 j}^{i}\left(\begin{array}{l}
n-r \\
i
\end{array}\right)\left(\begin{array}{l}
i \\
j
\end{array}\right) \frac{\Gamma\left(A_{1 \tau}\right)}{B_{1 \tau}^{A_{\tau} \tau}} \frac{\Gamma\left(A_{2 \tau}\right.}{B_{2 \tau}^{A_{2 \tau}}} \frac{\Gamma\left(A_{3 \tau}\right)}{B_{3 \tau}^{A_{3 \tau}}} B\left(B_{0 \tau}, C_{0 \tau}\right) B\left(A_{0 \tau}+2, B_{0 \tau}+C_{0 \tau}\right)\right\} \\
& -2\left\{Q_{\tau}^{-1} \sum_{i=0}^{n-r} \sum_{j=0}^{i}\left(\begin{array}{l}
n-r \\
i
\end{array}\right)\left(\begin{array}{l}
i \\
j
\end{array}\right) \frac{\Gamma\left(A_{1 \tau}\right)}{B_{1 \tau}^{A_{t}}} \frac{\Gamma\left(A_{2 \tau}\right)}{\left.B_{2 \tau}\right)} \frac{\Gamma\left(A_{3 \tau}\right)}{B_{3 \tau}^{A_{\tau}}} B\left(B_{0 \tau}, C_{0 \tau}\right) B\left(A_{0 \tau}+1, B_{0 \tau}+C_{0 \tau}\right)\right\}
\end{aligned}
$$

$$
\begin{aligned}
& \rho\left(\hat{p}_{2}\right)=2\left\{Q_{\tau}^{-1} \sum_{i=0}^{n-r} \sum_{=0 j}^{i}\left(\begin{array}{l}
n-r \\
i
\end{array}\right)\left(\begin{array}{l}
i \\
j
\end{array}\right) \frac{\Gamma\left(A_{1 \tau}\right)}{B_{1 \tau}^{A_{t \tau}}} \frac{\Gamma\left(A_{2 \tau}\right)}{B_{2 \tau}^{A_{\tau}}} \frac{\Gamma\left(A_{3 \tau}\right)}{B_{3 \tau}^{A_{3 \tau}}} B\left(A_{0 \tau}, C_{0 \tau}\right) B\left(B_{0 \tau}+2, A_{0 \tau}+C_{0 \tau}\right)\right\}^{\frac{1}{2}} \\
& -2\left\{Q_{\tau}^{-1} \sum_{i=0}^{n-r} \sum_{j=0}^{i}\left(\begin{array}{l}
n-r \\
i
\end{array}\right)\left(\begin{array}{l}
i \\
j
\end{array}\right) \frac{\Gamma\left(A_{1 \tau}\right.}{B_{1 \tau}^{A_{\tau} \tau}} \frac{\Gamma\left(A_{2 \tau}\right)}{B_{2 \tau}^{A_{\tau}}} \frac{\Gamma\left(A_{3 \tau}\right)}{B_{3 \tau}^{A_{3 \tau}}} B\left(A_{0 \tau}, C_{0 \tau}\right) B\left(B_{0 \tau}+1, A_{0 \tau}+C_{0 \tau}\right)\right\}
\end{aligned}
$$

Bayes estimators and posterior risks using the UP, the JP and IP under DeGroot loss function (DLF)

DeGroot (2005) introduced the asymmetric loss function, $L(\beta, d)=\left(\frac{\beta-d}{d}\right)^{2}$ known as DeGroot loss function (DLF). The Bayes estimator and its posterior risk under DLF are: $\hat{d}=\frac{E_{\beta \mid x}\left(\beta^{2}\right)}{E_{\beta \mid x}(\beta)}$ and $\rho(\hat{d})=1-\frac{\left\{E_{\beta \mid x}(\beta)\right\}^{2}}{E_{\beta \mid x}\left(\beta^{2}\right)}$, respectively. The Bayes estimators and posterior risks using the UP, JP and IP for parameters $\beta_{1}, \beta_{2}, \beta_{3}, p_{1}$ and $p_{2}$ under DLF are:

$\hat{\beta}_{1 \tau}=\frac{\left\{Q_{\tau}^{-1} \sum_{i=0}^{n-r} \sum_{j=0}^{i}\left(\begin{array}{l}n-r \\ i\end{array}\right)\left(\begin{array}{l}i \\ j\end{array}\right) \frac{\Gamma\left(A_{1 \tau}+2\right)}{B_{1 \tau}^{A_{t \tau}+2}} \frac{\Gamma\left(A_{2 \tau}\right)}{B_{2 \tau}^{A_{\tau} \tau}} \frac{\Gamma\left(A_{3 \tau}\right)}{B_{3 \tau}^{A_{\tau}}} B\left(A_{0 \tau}, C_{0 \tau}\right) B\left(B_{0 \tau}, A_{0 \tau}+C_{0 \tau}\right)\right\}}{\left.\left\{Q_{\tau}^{-1} \sum_{i=0}^{n-r} \sum_{j=0}^{i}\left(\begin{array}{l}n-r \\ i\end{array}\right)\left(\begin{array}{l}i \\ j\end{array}\right) \frac{\Gamma\left(A_{1 \tau}+1\right)}{B_{1 \tau}^{A_{1 \tau}+1}} \frac{\Gamma\left(A_{2 \tau}\right)}{B_{2 \tau}}\right) \frac{\Gamma\left(A_{3 \tau}\right)}{B_{3 \tau} A_{\tau \tau}} B\left(A_{0 \tau}, C_{0 \tau}\right) B\left(B_{0 \tau}, A_{0 \tau}+C_{0 \tau}\right)\right\}}$

$\hat{\beta}_{2 \tau}=\frac{\left\{Q_{\tau}^{-1} \sum_{i=0}^{n-r} \sum_{j=0}^{i}\left(\begin{array}{l}n-r \\ i\end{array}\right)\left(\begin{array}{l}i \\ j\end{array}\right) \frac{\Gamma\left(A_{1 \tau}\right)}{B_{1 \tau}^{A_{1 \tau}}} \frac{\Gamma\left(A_{2 \tau}+2\right)}{B_{2 \tau}^{A_{2}+2}} \frac{\Gamma\left(A_{3 \tau}\right)}{B_{3 \tau}^{A_{3 \tau}}} B\left(A_{0 \tau}, C_{0 \tau}\right) B\left(B_{0 \tau}, A_{0 \tau}+C_{0 \tau}\right)\right\}}{\left\{Q_{\tau}^{-1} \sum_{i=0}^{n-r} \sum_{j=0}^{i}\left(\begin{array}{l}n-r \\ i\end{array}\right)\left(\begin{array}{l}i \\ j\end{array}\right) \frac{\Gamma\left(A_{1 \tau}\right)}{B_{1 \tau}^{A_{t \tau}}} \frac{\Gamma\left(A_{2 \tau}+1\right)}{B_{2 \tau}^{A_{\tau}+1}} \frac{\Gamma\left(A_{3 \tau}\right)}{B_{3 \tau}^{A_{3 \tau}}} B\left(A_{0 \tau}, C_{0 \tau}\right) B\left(B_{0 \tau}, A_{0 \tau}+C_{0 \tau}\right)\right\}}$

$\hat{\beta}_{3 \tau}=\frac{\left\{Q_{\tau}^{-1} \sum_{i=0}^{n-r} \sum_{j=0}^{i}\left(\begin{array}{l}n-r \\ i\end{array}\right)\left(\begin{array}{l}i \\ j\end{array}\right) \frac{\Gamma\left(A_{1 \tau}\right)}{\left.B_{1 \tau}^{A_{\tau}}\right)} \frac{\Gamma\left(A_{2 \tau}\right)}{B_{2 \tau}^{A_{2 \tau}}} \frac{\Gamma\left(A_{3 \tau}+2\right)}{B_{3 \tau}^{A_{\tau}+2}} B\left(A_{0 \tau}, C_{0 \tau}\right) B\left(B_{0 \tau}, A_{0 \tau}+C_{0 \tau}\right)\right\}}{\left\{Q_{\tau}^{-1} \sum_{i=0}^{n-r} \sum_{j=0}^{i}\left(\begin{array}{l}n-r \\ i\end{array}\right)\left(\begin{array}{l}i \\ j\end{array}\right) \frac{\Gamma\left(A_{1 \tau}\right)}{\left.B_{1 \tau}\right)} \frac{\Gamma\left(A_{\tau \tau}\right)}{\left.B_{2 \tau}^{A_{\tau}}\right)} \frac{\Gamma\left(A_{3 \tau}+1\right)}{B_{3 \tau}^{A_{3 \tau}+1}} B\left(A_{0 \tau}, C_{0 \tau}\right) B\left(B_{0 \tau}, A_{0 \tau}+C_{0 \tau}\right)\right\}}$ 


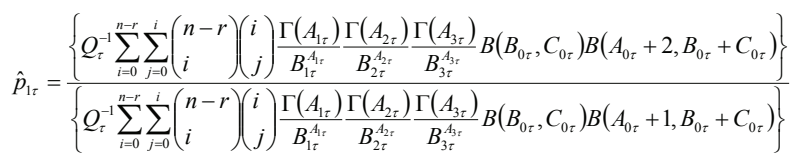

$\hat{p}_{2 \tau}=\frac{\left\{Q_{\tau}^{-1} \sum_{i=0}^{n-r} \sum_{j=0}^{i}\left(\begin{array}{l}n-r \\ i\end{array}\right)\left(\begin{array}{l}i \\ j\end{array}\right) \frac{\Gamma\left(A_{1 \tau}\right)}{\left.B_{1 \tau}^{A_{\tau}}\right)} \frac{\Gamma\left(A_{2 \tau}\right)}{\left.B_{2 \tau}^{A_{2 \tau}}\right)} \frac{\Gamma\left(A_{3 \tau}\right)}{B_{3 \tau}^{A_{3 \tau}}} B\left(A_{0 \tau}, C_{0 \tau}\right) B\left(B_{0 \tau}+2, A_{0 \tau}+C_{0 \tau}\right)\right\}}{\left\{Q_{\tau}^{-1} \sum_{i=0}^{n-r} \sum_{j=0}^{i}\left(\begin{array}{l}n-r \\ i\end{array}\right)\left(\begin{array}{l}i \\ j\end{array}\right) \frac{\Gamma\left(A_{1 \tau}\right)}{\left.B_{1 \tau}^{A}\right)} \frac{\Gamma\left(A_{2 \tau}\right)}{\left.B_{2 \tau}^{A_{\tau}}\right)} \frac{\Gamma\left(A_{3 \tau}\right)}{B_{3 \tau}^{A_{\tau}}} B\left(A_{0 \tau}, C_{0 \tau}\right) B\left(B_{0 \tau}+1, A_{0 \tau}+C_{0 \tau}\right)\right\}}$

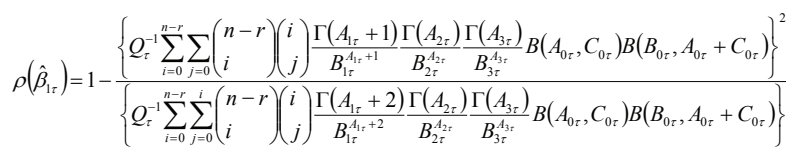

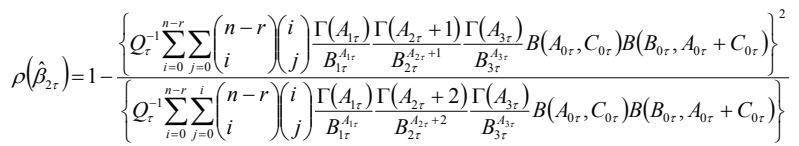

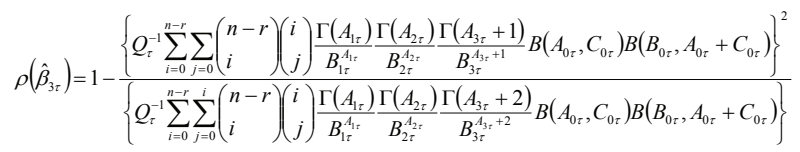

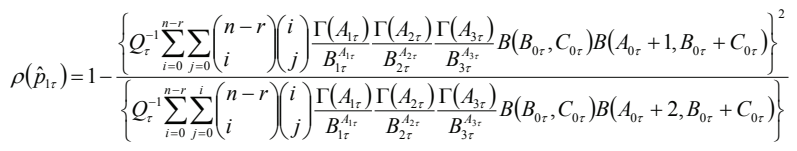

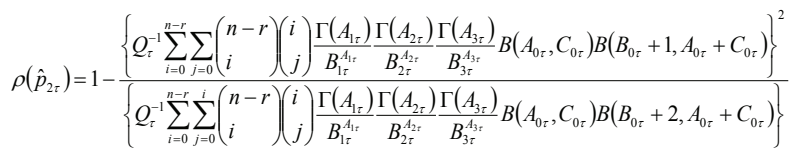

\section{Elicitation of hyperparameters}

Elicitation is the key task for subjective Bayesian. The whole procedure for quantifying the prior information in the form of prior distribution is precisely known as elicitation. Aslam (2003) proposed different methods of elicitation based on prior predictive distribution for the elicitation of hyperparameters. In this study, we use the method of elicitation using prior predictive distribution based on predictive probabilities. In this method, confidence levels of the prior predictive are obtained for the particular intervals of the random variables. The set of hyperparameters, for which the difference between the elicited probabilities and the expert predictive probabilities is minimum, is considered.

\section{Elicitation of hyperparameters using the gamma prior}

For eliciting the hyperparameters, prior predictive distribution (PPD) is used. The PPD for a random variable $y$ is:

$$
\begin{aligned}
& p(y)=\int_{\psi} p(y \mid \psi) \pi_{3}(\psi) d \psi \\
& p(y)=\frac{1}{(a+b+c)}\left[\frac{a a_{1} b_{1}^{a_{1}} y^{-2}}{\left(b_{1}+y^{-1}\right)^{a_{1}+1}}+\frac{b a_{2} b_{2}^{a_{2}} y^{-2}}{\left(b_{2}+y^{-1}\right)^{a_{2}+1}}+\frac{c a_{3} b_{3}^{a_{3}} y^{-2}}{\left(b_{3}+y^{-1}\right)^{a}+1}\right]
\end{aligned}
$$

We choose the prior predictive probabilities satisfying the laws of probability, to elicit the hyperparameters of the prior density. Using the prior predictive distribution, we consider the nine intervals $(0,1),(1,2),(2,3),(3,4)$, $(4,5),(5,6),(6,7),(7,8)$ and $(8,9)$ with probabilities $0.67,0.13,0.06,0.03,0.02,0.01,0.01,0.008$ and 0.006 , respectively given an expert opinion.

For eliciting the hyperparameters $a_{1}, a_{2}, a_{3}, b_{1}, b_{2}$, $b_{3}, a, b$ and $c$, the equations are simultaneously solved through the computer programme developed in SAS package using the 'PROC SYSLIN' command; the values of the hyperparameters are found to be 1.7388 , $0.4722,0.3528,1.0779,0.1469,0.0737,0.3695,1.4360$ and 8.8749 , respectively.

\section{Limiting expressions}

Letting $t \rightarrow \infty$, all the observations that are incorporated in our analysis are uncensored and therefore, $r$ tends to $n$, $r_{1}$ tends to the unknown $n_{1}, r_{2}$ tends to the unknown $n_{2}$ and $r_{3}$ tends to the unknown $n_{3}$. As a result, the amount of information contained in the sample is increasing, which consequently results in the reduction of the variances of the estimates. The limiting (complete sample) expressions for Bayes estimators and posterior risks using the UP, JP and IP under SELF, PLF and DLF are given in Tables $1-4$. The properties of Bayes estimators and their corresponding posterior risks using complete sampling under SELF are given in Table VII in appendix. 
Table 1: Limiting expressions for the Bayes estimators as $t \rightarrow \infty$ using the UP, JP and GP under SELF, PLF and DLF

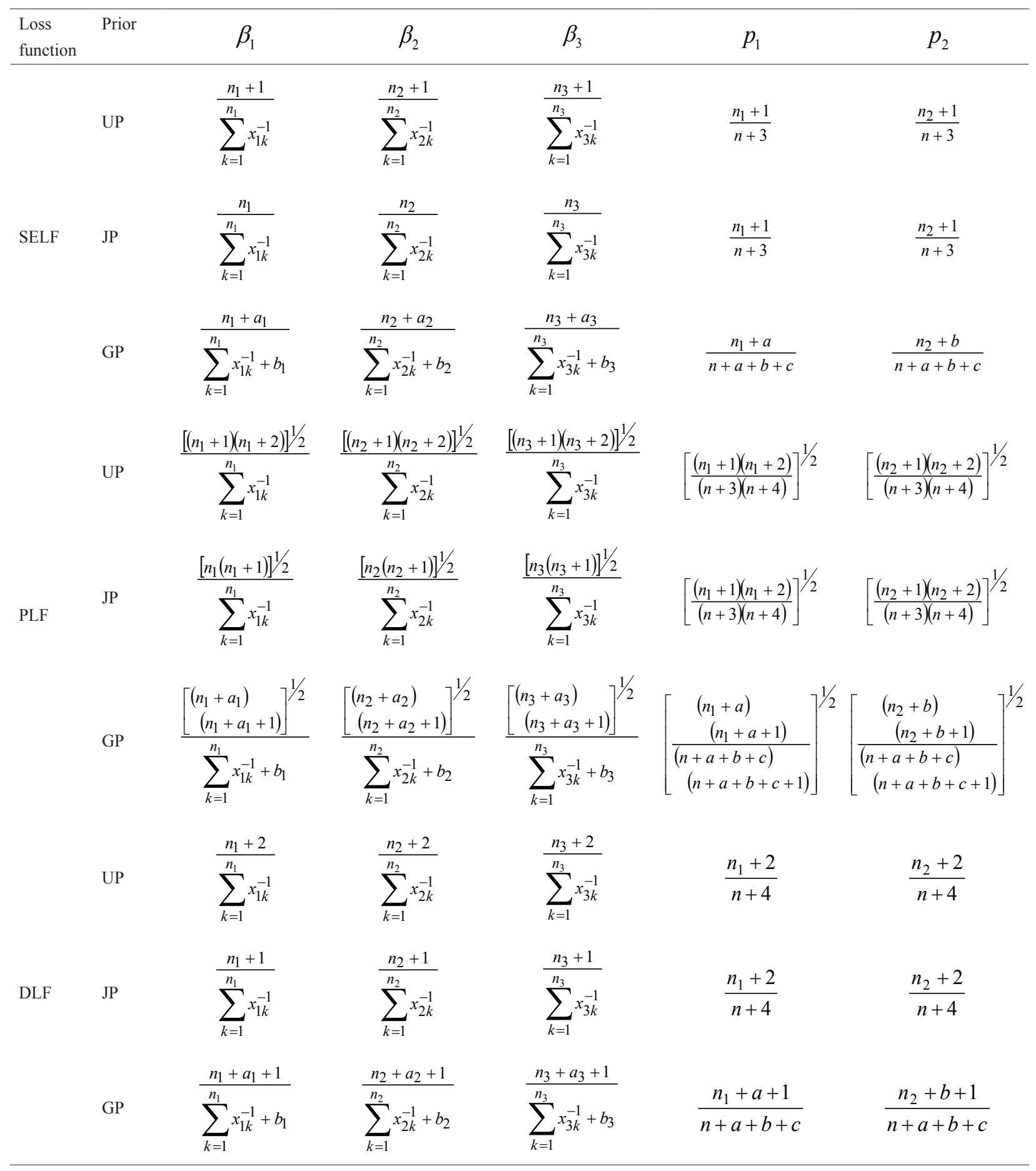


Table 2: Limiting expressions for the posterior risks as $t \rightarrow \infty$ using the UP, JP and GP under SELF

\begin{tabular}{cccc}
\hline Parameters & UP & JP & GP \\
\hline$\beta_{1}$ & $\frac{n_{1}+1}{\left(\sum_{k=1}^{n_{1}} x_{1 k}^{-1}\right)^{2}}$ & $\frac{n_{1}}{\left(\sum_{k=1}^{n_{1}} x_{1 k}^{-1}\right)^{2}}$ & $\left(\sum_{k=1}^{n_{1}} x_{1 k}^{-1}+b_{1}\right)^{2}$ \\
$\beta_{2}$ & $\frac{n_{2}+1}{\left(\sum_{k=1}^{n_{2}} x_{2 k}^{-1}\right)^{2}}$ & $\frac{n_{2}}{\left(\sum_{k=1}^{n_{2}} x_{2 k}^{-1}\right)^{2}}$ & $\frac{\left(\sum_{k=1}^{n_{2}} x_{2 k}^{-1}+b_{2}\right)^{2}}{\left(\sum_{k=1}^{n_{3}} x_{3 k}^{-1}\right)^{2}}$ \\
$\beta_{3}$ & $\frac{\left(n_{1}+1\right)\left(n_{2}+n_{3}+2\right)}{(n+3)^{2}(n+4)}$ & $\frac{\left(n_{3} x_{3 k}^{-1}\right)^{2}}{(n+1)\left(n_{2}+n_{3}+2\right)}$ & $\frac{\left(n_{k} x_{3 k}^{-1}+b_{3}\right)^{2}}{(n+a+a)\left(n_{2}+n_{3}+b+c\right)}$ \\
$p_{1}$ & $\frac{\left(n_{2}+1\right)\left(n_{1}+n_{3}+2\right)}{(n+3)^{2}(n+4)}$ & $\frac{\left(n_{2}+1\right)\left(n_{1}+n_{3}+2\right)}{(n+3)^{2}(n+4)}$ & $\frac{\left(n_{2}+b\right)\left(n_{1}+n_{3}+a+c\right)}{(n+a+b+c)^{2}(n+a+b+c+1)}$ \\
$p_{2}$ & &
\end{tabular}

Table 3: Limiting expressions for the posterior risks as $t \rightarrow \infty$ using the UP, JP and GP under PLF

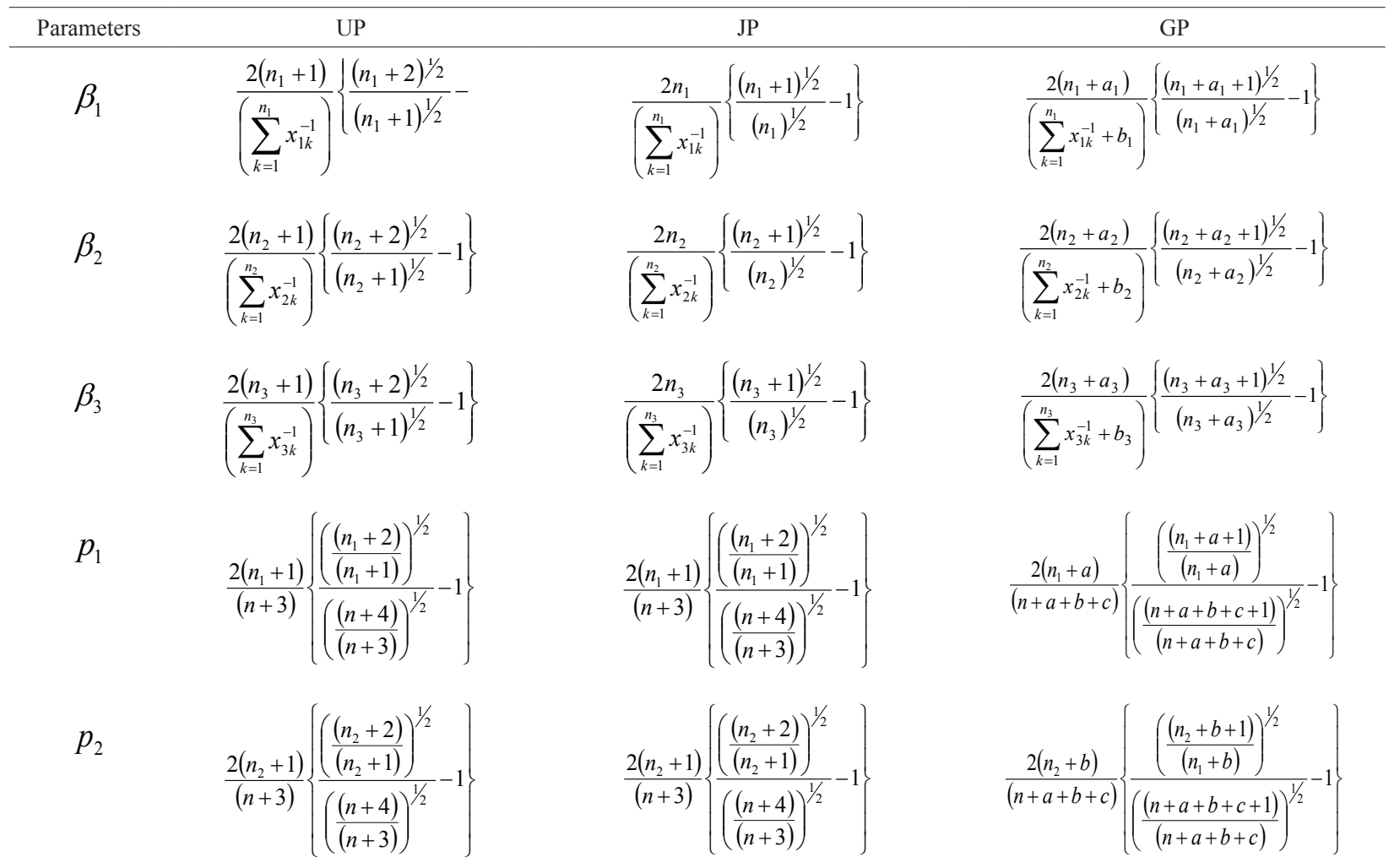


Table 4: Limiting expressions for the posterior risks as $t \rightarrow \infty$ using the UP, JP and GP under DLF

\begin{tabular}{cccc}
\hline Parameters & UP & JP & Gamma prior \\
\hline$\beta_{1}$ & $\frac{1}{n_{1}+2}$ & $\frac{1}{n_{1}+1}$ & $\frac{1}{n_{1}+a_{1}+1}$ \\
$\beta_{2}$ & $\frac{1}{n_{2}+2}$ & $\frac{1}{n_{2}+1}$ & $\frac{1}{n_{2}+a_{2}+\ldots 1}$ \\
$\beta_{3}$ & $\frac{1}{n_{3}+2}$ & $\frac{1}{n_{3}+1}$ & $\frac{1}{n_{3}+a_{3}+1}$ \\
$p_{1}$ & $\frac{\left(n_{2}+n_{3}+2\right)}{\left(n_{1}+2\right)(n+3)}$ & $\frac{\left(n_{2}+n_{3}+2\right)}{\left(n_{1}+2\right)(n+3)}$ & $\frac{\left(n_{2}+n_{3}+b+c\right)}{\left(n_{1}+a+1\right)(n+a+b+c)}$ \\
$p_{2}$ & $\frac{\left(n_{1}+n_{3}+2\right)}{\left(n_{2}+2\right)(n+3)}$ & $\frac{\left(n_{1}+n_{3}+2\right)}{\left(n_{2}+2\right)(n+3)}$ & $\frac{\left(n_{1}+n_{3}+a+c\right)}{\left(n_{2}+b+1\right)(n+a+b+c)}$ \\
\hline
\end{tabular}

Table 5: Bayes estimates (BEs) and posterior risks (PRs) of 3-component mixture of Gumbel type-II distributions using the UP, JP, and GP under SELF, PLF and DLF with Crowder et al. (1994) mixture data

\begin{tabular}{|c|c|c|c|c|c|c|c|}
\hline Prior & Loss & functions & $\hat{\beta}_{1}$ & $\hat{\beta}_{2}$ & $\hat{\beta}_{3}$ & $\hat{p}_{1}$ & $\hat{p}_{2}$ \\
\hline \multirow{6}{*}{ UP } & \multirow{2}{*}{ SELF } & $\mathrm{BE}$ & 3.00174 & 2.43043 & 1.99007 & 0.19558 & 0.34294 \\
\hline & & PR & 0.676233 & 0.247192 & 0.121833 & 0.002011 & 0.002962 \\
\hline & \multirow{2}{*}{ PLF } & $\mathrm{BE}$ & 3.11234 & 2.48076 & 2.02045 & 0.20066 & 0.34723 \\
\hline & & PR & 0.221205 & 0.100665 & 0.060756 & 0.010148 & 0.008582 \\
\hline & \multirow{2}{*}{ DLF } & $\mathrm{BE}$ & 3.22702 & 2.53213 & 2.05129 & 0.20586 & 0.35158 \\
\hline & & PR & 0.069811 & 0.040167 & 0.029845 & 0.049934 & 0.024564 \\
\hline \multirow{6}{*}{ JP } & \multirow{2}{*}{ SELF } & $\mathrm{BE}$ & 2.79327 & 2.34029 & 1.93776 & 0.19715 & 0.34295 \\
\hline & & PR & 0.620029 & 0.236873 & 0.118651 & 0.002034 & 0.002956 \\
\hline & \multirow{2}{*}{ PLF } & $\mathrm{BE}$ & 2.90213 & 2.39036 & 1.96814 & 0.20225 & 0.34723 \\
\hline & & PR & 0.217730 & 0.100144 & 0.060755 & 0.010184 & 0.008567 \\
\hline & \multirow{2}{*}{ DLF } & $\mathrm{BE}$ & 3.01524 & 2.44151 & 1.99899 & 0.20747 & 0.35157 \\
\hline & & PR & 0.073617 & 0.041456 & 0.030631 & 0.049718 & 0.024520 \\
\hline \multirow{6}{*}{ GP } & \multirow{2}{*}{ SELF } & $\mathrm{BE}$ & 2.63492 & 2.40087 & 1.91168 & 0.17412 & 0.31509 \\
\hline & & PR & 0.469892 & 0.242927 & 0.113700 & 0.001672 & 0.002551 \\
\hline & \multirow{2}{*}{ PLF } & $\mathrm{BE}$ & 2.72262 & 2.45094 & 1.94119 & 0.17886 & 0.31911 \\
\hline & & PR & 0.175413 & 0.100138 & 0.059021 & 0.009473 & 0.008045 \\
\hline & \multirow{2}{*}{ DLF } & $\mathrm{BE}$ & 2.81325 & 2.50206 & 1.97115 & 0.18372 & 0.32319 \\
\hline & & PR & 0.063390 & 0.040440 & 0.030173 & 0.052265 & 0.025053 \\
\hline
\end{tabular}




\section{RESULTS AND DISCUSSION}

In order to obtain and investigate the properties of the derived estimators, simulation analysis has been carried out. Simulation study is a flexible methodology to illustrate the properties of the Bayes estimators of the 3-component mixture of the Gumbel type-II distributions using the UP, JP and GP under SELF, PLF and DLF in terms of different sample sizes and test termination times. Random samples of size $n=50,100,200$ and 300 have been generated from the Gumbel type-II mixture model by using different values of the vector of the parameters $=\{(2,3,4,0.20,0.60),(3,3,3,0.40,0.40)\}$.

For a fixed sample size, test termination time and set of parameters, the observations are randomly taken from the first, second and third component density. The observations, which are greater than the fixed ' $t$ ' were

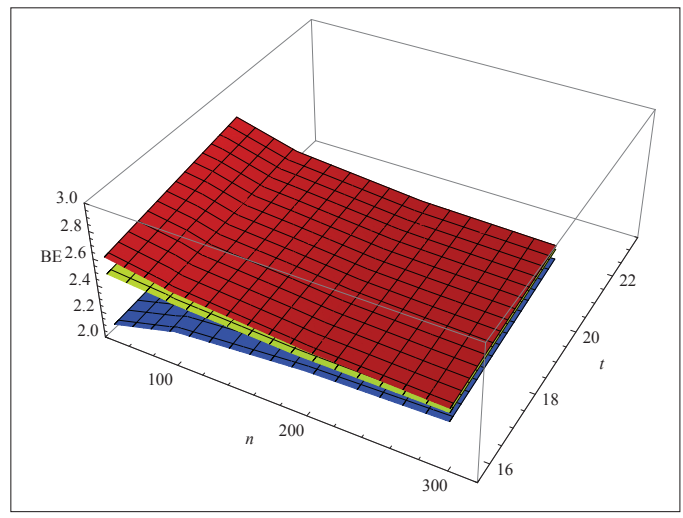

Figure 1: $\quad$ BEs for $\beta_{1}$ under SELF

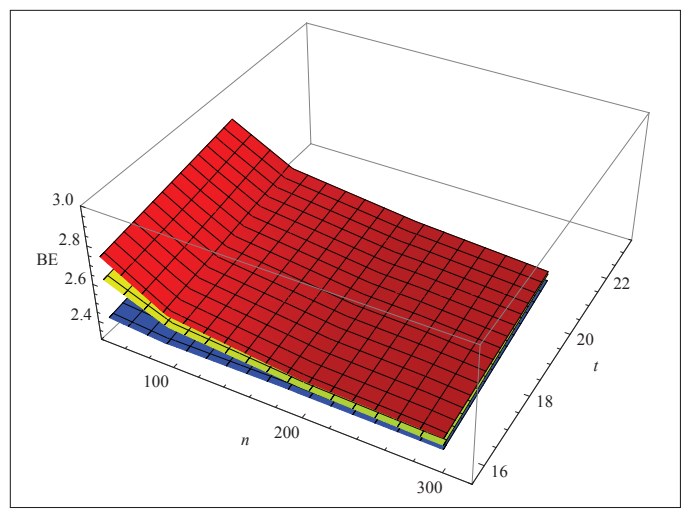

Figure 3: $\quad$ BEs for $\beta_{2}$ under PLF declared as censored observations. For each t, only failures were identified either as a member of subpopulation I, subpopulation II or subpopulation III. This process was replicated 1000 times using a Mathematica 10.0 programme. For each of the 1000 samples, the Bayes estimators and posterior risks were computed and the results are presented in the appendix from Tables I to VII. The simulation study provides some interesting properties of the Bayes estimates. The properties are highlighted in terms of sample sizes, the size of mixing proportions parameters, different loss functions and censoring rates. Estimates are obtained by using the UP, JP and GP. The same results have been examined in Figures $1-10$ that are based on simulation analysis tables corresponding to the different prior distributions and various loss functions. In Figures $1-4$, the UP, JP and GP are represented by red, yellow and blue colours, while in Figures 5 - 10, SELF, PLF and DLF are represented by red, yellow and blue colours, respectively.

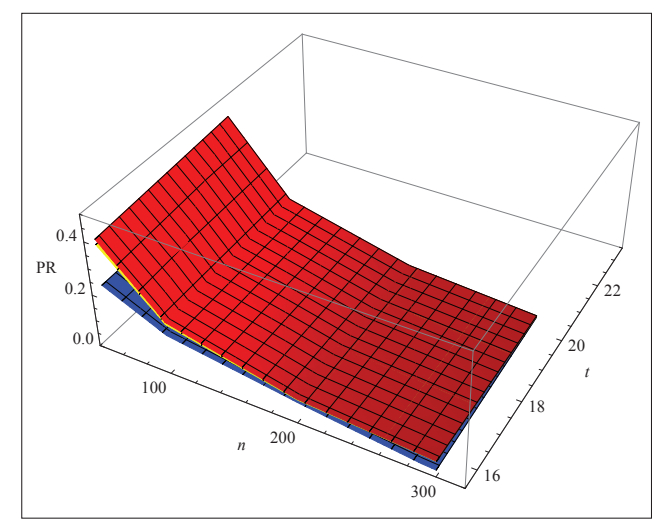

Figure 2: $\quad$ PRs for $\beta_{1}$ under SELF

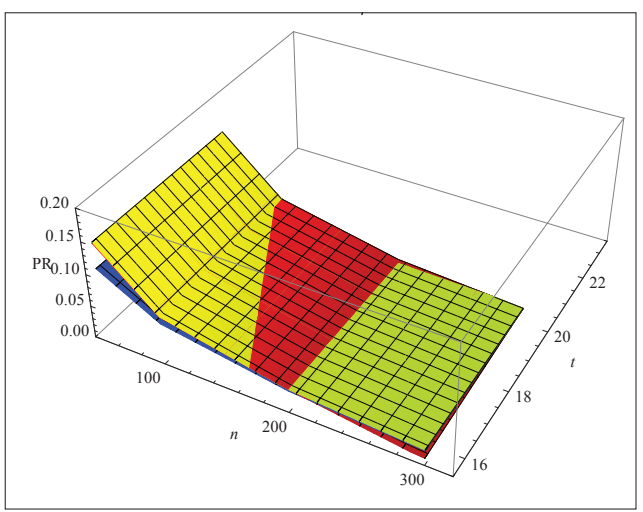

Figure 4: $\quad$ PRs for $\beta_{2}$ under PLF 


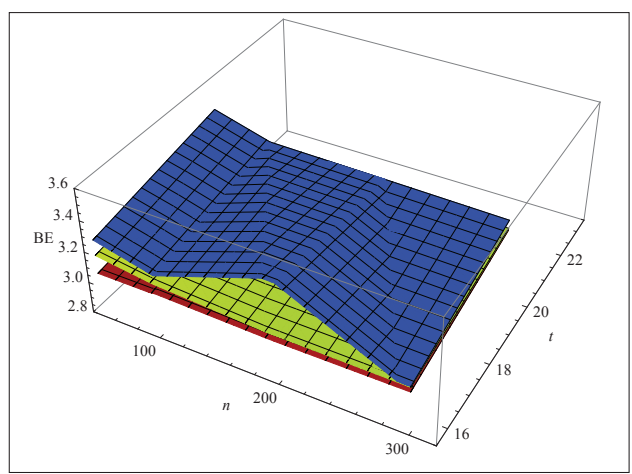

Figure 5: $\quad$ BEs for $\beta_{3}$ using GP

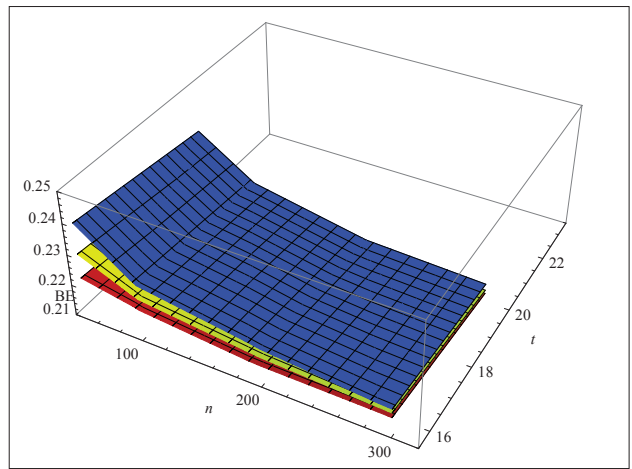

Figure 7: BEs for $P_{1}$ using UP

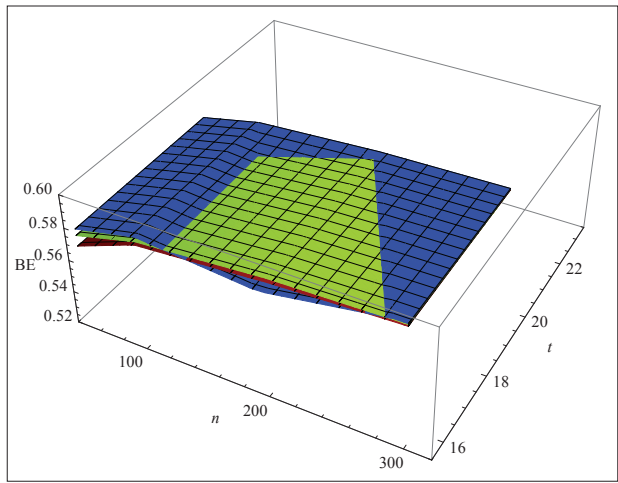

Figure 9: $\quad$ BEs for $P_{2}$ using GP

It is observed that the PRs of Bayes estimators using different priors and loss functions reduce with an increase in sample size at different test termination times. For a smaller test termination time, the PRs of Bayes estimators are larger than the PRs for a larger test termination time, irrespective of the priors, loss function and sample size. Also, the PRs of Bayes estimators are smaller (larger) for

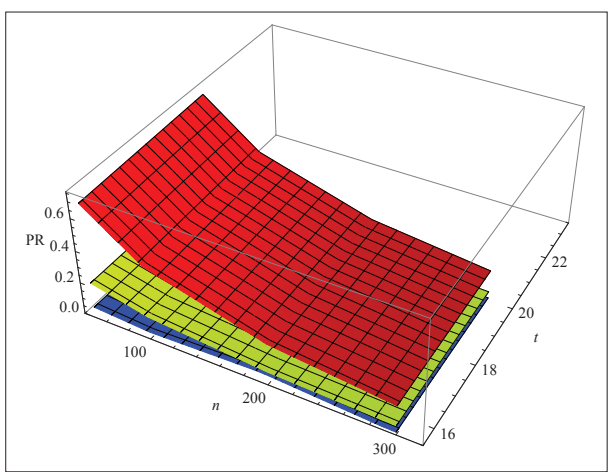

Figure 6: $\quad$ PRs for $\beta_{3}$ using GP

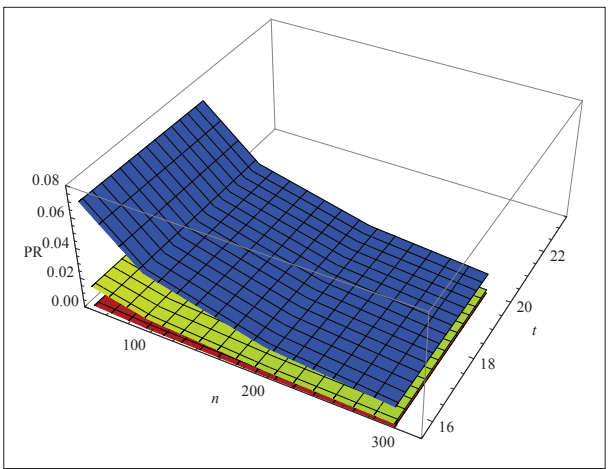

Figure 8: $\quad$ PRs for $P_{1}$ using UP

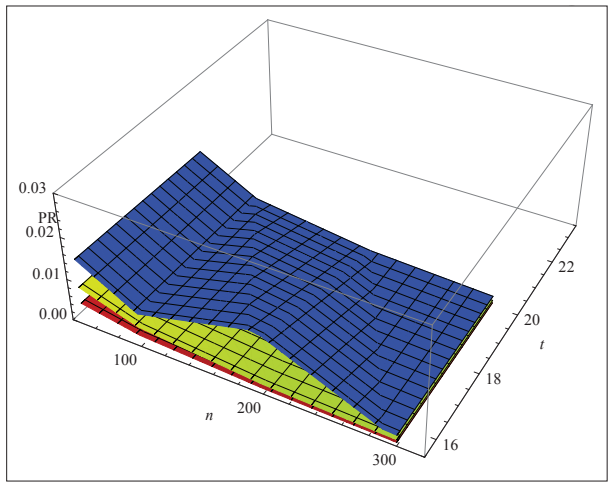

Figure 10: PRs for $P_{2}$ using GP

smaller (larger) component parametric values for each sample size and test termination time considered in the simulation study.

In Table VII in appendix, some results for the complete sample under SELF are given, which generally highlights the importance of incorporating full information into the 
sample by producing Bayes estimates that do not involve underestimation for any loss function or prior used, and provides smaller posterior risks. The same results have been obtained under PLF and DLF.

\section{A real life data application}

Crowder et al. (1994) have reported the data on fibre failure strength, and the breaking strength of fibre sections of lengths 5, 12, 30 and $45 \mathrm{~mm}$. To elucidate the proposed methodology, we have taken the data on 3-component, namely, 5, 12 and $30 \mathrm{~mm}$, respectively. The values are right censored at 4.0 i.e. $\mathrm{t}=4.0$. The sample statistics required to evaluate the proposed estimates are as follows:

$$
\begin{aligned}
& n=102, r_{1}=15, r_{2}=26, r_{3}=34, r=75, n-r=27, \\
& \sum_{k=1}^{r_{1}} x_{1 k}^{-1}=4.2612, \sum_{k=1}^{r_{2}} x_{2 k}^{-1}=8.9087, \sum_{k=1}^{r_{3}} x_{3 k}^{-1}=14.2608 .
\end{aligned}
$$

Bayes estimates and Bayes posterior risks (BPRs) using the UP, JP and GP under SELF, PLF and DLF are given in Table 5.

It is noted that the results gained from real data are compatible with simulation results. The results declare that the execution of the informative prior is better than the non-informative priors. It is also examined that execution of DLF is preferred for estimating the component parameters, while SELF is better for estimating the proportion parameters.

\section{CONCLUSION}

As far as the problem of selecting the most suitable prior is concerned, it can be seen that the informative prior (GP) is the best prior among the assumed priors. On the other hand, DLF is observed performing better than PLF and SELF for estimating component parameters, whereas for estimating proportion parameters, SELF is observed superior to PLF and DLF. Therefore, the informative prior has a clear edge over the non-informative prior. It is to be noted that the selection of the best prior (loss function) for a given loss function (prior) is made on PRs associated with it. Also, the selection of the best prior and loss function does not depend on the sample size and test termination time. The findings of this study are compatible with the findings described by Feroz and Aslam (2012) in Bayesian estimation of 2-component mixture of Gumbel Type-II distributions and are useful for analysts looking to model lifetime data, where the causes of failure are more than one.
From simulated results, we observed that an increase in the sample size and test termination time provides better Bayes estimators. Furthermore, as the sample size increases (decreases) the posterior risks of Bayes estimators decreases (increases) for a fixed test termination time. Also, the DLF is observed as a suitable choice for estimating component parameters, and SELF is preferable for estimating proportion parameters. Finally, we conclude that the GP is the suitable prior in order to estimate the component parameters. When SELF is used, the GP is an appropriate prior for proportion parameters. The same pattern is observed for JP when non-informativse priors are considered.

\section{REFERENCES}

1. Abbas K., Fu J. \& Tang Y. (2013). Bayesian estimation of gumbel type-II distribution. Data Science Journal 12: $33-46$.

DOI: https://doi.org/10.2481/dsj.13-022

2. Ali S. (2015). Mixture of the inverse rayleigh distribution: properties and estimation in a bayesian framework. Applied Mathematical Modeling 39(2): 515 - 530. DOI: https://doi.org/10.1016/j.apm.2014.05.039

3. Aslam M. (2003). An application of prior predictive distribution to elicit the prior density. Journal of Statistical Theory and Applications 2(1): $70-83$.

4. Berger J.O. (1985). Statistical Decision Theory and Bayesian Analysis. Springer-Verlag, New York, USA.

5. Chen K.W., Papadopoulos A.S. \& Tamer P. (1989). On Bayes estimation for mixtures of two Weibull distributions under type I censoring. Microelectronics Reliability 29(4): $609-617$.

6. Crowder M.J., Kimber A.C., Smith R.L. \& Sweeting T.J. (1994). Statistical Analysis of Reliability Data. CRC Press, Boca Raton, USA.

7. DeGroot M.H. (2005). Optimal Statistical Decisions. John Wiley and Sons, Inc., New Jersey, USA.

DOI: https://doi.org/10.2307/2533242

8. Feroze N. \& Aslam M. (2012). Bayesian analysis of gumbel type II distribution under doubly censored samples using different loss functions. Caspian Journal of Applied Sciences Research 1(10): $1-10$.

9. Feroze N. \& Aslam M. (2013). Bayesian estimation of two-component mixture of gumbel type II distribution under informative priors. International Journal of Advanced Science and Technology 53: 11 - 30.

10. Gumbel E. (1958). Statistics of Extremes. Columbia University Press, New York, USA.

11. Jeffreys H. (1946). An invariant form for the prior probability in estimation problems. Proceedings of the Royal Society of London A: Mathematical, Physical and Engineering Sciences 186(1007): 453 - 461. DOI: https://doi.org/10.1098/rspa.1946.0056

12. Jeffreys H. (1961). Theory of Probability. Oxford University Press, New York, USA. 
13. Kazmi S., Aslam M. \& Ali S. (2012). On the bayesian estimation for two component mixture of maxwell distribution, assuming type I censored data. International Journal of Applied Science and Technology 2(1): 197 218.

14. Kotz S. \& Nadarajah S. (2000). Extreme Value Distributions: Theory and Applications. World Scientific Publishing, Singapore.

DOI: https://doi.org/10.1142/p191

15. Legendre A.M. (1805). Nouvelles Méthodes Pour la Détermination des Orbites des Comètes (no. 1). F. Didot, Paris, France.

16. Mendenhall W. \& Hader R. (1958). Estimation of parameters of mixed exponentially distributed failure time distributions from censored life test data. Biometrika 45(3 - 4): $504-520$.

17. Noor F. \& Aslam M. (2013). Bayesian inference of the inverse weibull mixture distribution using type-I censoring. Journal of Applied Statistics 40(5): 1076 - 1089.
DOI: https://doi.org/10.1080/02664763.2013.780157

18. Norstrøm J.G. (1996). The use of precautionary loss functions in risk analysis. IEEE Transactions on Reliability 45(3): $400-403$.

DOI: https://doi.org/10.1109/24.536992

19. Reyad H. \& Ahmed S.O. (2015). E-Bayesian analysis of the gumbel type-II distribution under type-II censored scheme. International Journal of Advanced Mathematical Sciences 3(2): 108 - 120.

DOI: https://doi.org/10.14419/ijams.v3i2.5093

20. Saleem M., Aslam M. \& Economou P. (2010). On the bayesian analysis of the mixture of power function distribution using the complete and the censored sample. Journal of Applied Statistics 37(1): 25 - 40. DOI: https://doi.org/10.1080/02664760902914557

21. Salinas V., Perez P., Gonzalez E. \& Vaquera H. (2012). Goodness of fit tests for the gumbel distribution with type II right censored data. Revista Colombiana de Estadística 35(3): $409-424$. 


\section{APPENDIX}

Table I: Bayes estimates (BEs) and posterior risks (PRs) of 3-component mixture of Gumbel type-II distributions using the UP under SELF, PLF and DLF with $\beta_{1}=2, \beta_{2}=3, \beta_{3}=4, p_{1}=0.20, p_{2}=0.60, t=15,20$

\begin{tabular}{|c|c|c|c|c|c|c|c|c|}
\hline \multirow[t]{2}{*}{$\mathrm{t}$} & \multirow[t]{2}{*}{$\mathrm{n}$} & \multirow{2}{*}{\multicolumn{2}{|c|}{ Loss functions }} & \multicolumn{5}{|c|}{ UP } \\
\hline & & & & $\hat{\beta}_{1}$ & $\hat{\beta}_{2}$ & $\hat{\beta}_{3}$ & $\hat{p}_{1}$ & $\hat{p}_{2}$ \\
\hline \multirow[t]{24}{*}{15} & \multirow[t]{6}{*}{50} & \multirow[t]{2}{*}{ SELF } & $\mathrm{BE}$ & 2.05963 & 2.55289 & 3.67339 & 0.22425 & 0.58263 \\
\hline & & & PR & 0.482935 & 0.264136 & 1.79543 & 0.003897 & 0.005405 \\
\hline & & \multirow[t]{2}{*}{ PLF } & $\mathrm{BE}$ & 2.19242 & 2.63541 & 3.91421 & 0.23213 & 0.58712 \\
\hline & & & PR & 0.214497 & 0.101558 & 0.437589 & 0.017182 & 0.009336 \\
\hline & & \multirow[t]{2}{*}{ DLF } & $\mathrm{BE}$ & 2.28623 & 2.67729 & 4.14929 & 0.24211 & 0.59072 \\
\hline & & & PR & 0.094131 & 0.038059 & 0.107369 & 0.072757 & 0.015954 \\
\hline & \multirow[t]{6}{*}{100} & \multirow[t]{2}{*}{ SELF } & $\mathrm{BE}$ & 1.9083 & 2.47895 & 3.37552 & 0.22084 & 0.58924 \\
\hline & & & PR & 0.207845 & 0.12508 & 0.748593 & 0.002019 & 0.002821 \\
\hline & & \multirow[t]{2}{*}{ PLF } & $\mathrm{BE}$ & 1.94512 & 2.52087 & 3.4678 & 0.22440 & 0.59252 \\
\hline & & & PR & 0.102426 & 0.049940 & 0.208573 & 0.009078 & 0.004782 \\
\hline & & \multirow[t]{2}{*}{ DLF } & $\mathrm{BE}$ & 2.0144 & 2.56464 & 3.55651 & 0.22876 & 0.59437 \\
\hline & & & PR & 0.051802 & 0.019695 & 0.058730 & 0.040271 & 0.008087 \\
\hline & \multirow[t]{6}{*}{200} & \multirow[t]{2}{*}{ SELF } & $\mathrm{BE}$ & 1.8058 & 2.45322 & 3.17286 & 0.21748 & 0.59484 \\
\hline & & & PR & 0.093528 & 0.061420 & 0.331186 & 0.001024 & 0.001441 \\
\hline & & \multirow[t]{2}{*}{ PLF } & $\mathrm{BE}$ & 1.84137 & 2.4676 & 3.22743 & 0.22039 & 0.59524 \\
\hline & & & PR & 0.050347 & 0.024795 & 0.101088 & 0.004668 & 0.002420 \\
\hline & & \multirow[t]{2}{*}{ DLF } & $\mathrm{BE}$ & 1.85144 & 2.48367 & 3.28593 & 0.22325 & 0.59672 \\
\hline & & & PR & 0.027120 & 0.010030 & 0.031212 & 0.0211 & 0.004069 \\
\hline & \multirow[t]{6}{*}{300} & \multirow[t]{2}{*}{ SELF } & $\mathrm{BE}$ & 1.77416 & 2.43709 & 3.11848 & 0.21716 & 0.59595 \\
\hline & & & PR & 0.059966 & 0.040370 & 0.212681 & 0.000686 & 0.000965 \\
\hline & & \multirow[t]{2}{*}{ PLF } & $\mathrm{BE}$ & 1.78096 & 2.44789 & 3.17044 & 0.21873 & 0.59607 \\
\hline & & & PR & 0.033017 & 0.016504 & 0.067174 & 0.003149 & 0.001623 \\
\hline & & DLF & $\mathrm{BE}$ & 1.80725 & 2.46302 & 3.18745 & 0.21992 & 0.59787 \\
\hline & & & PR & 0.018491 & 0.006724 & 0.021136 & 0.014418 & 0.002714 \\
\hline 20 & 50 & SELF & $\mathrm{BE}$ & 2.19255 & 2.75901 & 3.98405 & 0.22086 & 0.58229 \\
\hline & & & PR & 0.529995 & 0.294373 & 1.96752 & 0.003677 & 0.005170 \\
\hline & & PLF & $\mathrm{BE}$ & 2.29534 & 2.81129 & 4.16831 & 0.22801 & 0.58747 \\
\hline & & & PR & 0.215926 & 0.102638 & 0.434676 & 0.016384 & 0.008858 \\
\hline & & DLF & $\mathrm{BE}$ & 2.37214 & 2.84112 & 4.41512 & 0.23687 & 0.59125 \\
\hline & & & PR & 0.090964 & 0.036132 & 0.100275 & 0.070849 & 0.015106 \\
\hline & 100 & SELF & $\mathrm{BE}$ & 1.97456 & 2.62072 & 3.63898 & 0.21549 & 0.59029 \\
\hline & & & PR & 0.216296 & 0.132854 & 0.806272 & 0.001883 & 0.002682 \\
\hline & & PLF & $\mathrm{BE}$ & 2.00635 & 2.65615 & 3.68027 & 0.21964 & 0.59295 \\
\hline & & & PR & 0.101846 & 0.049947 & 0.20561 & 0.008666 & 0.004541 \\
\hline & & DLF & $\mathrm{BE}$ & 2.08985 & 2.70458 & 3.80788 & 0.22497 & 0.59449 \\
\hline & & & PR & 0.050019 & 0.018781 & 0.055241 & 0.039135 & 0.007705 \\
\hline & 200 & SELF & $\mathrm{BE}$ & 1.88328 & 2.60844 & 3.39904 & 0.21392 & 0.59515 \\
\hline & & & PR & 0.097850 & 0.065837 & 0.352952 & 0.000958 & 0.001367 \\
\hline & & PLF & $\mathrm{BE}$ & 1.91467 & 2.60709 & 3.4868 & 0.21558 & 0.59594 \\
\hline & & & PR & 0.050685 & 0.024890 & 0.101762 & 0.004461 & 0.002299 \\
\hline & & DLF & $\mathrm{BE}$ & 1.9436 & 2.62831 & 3.51094 & 0.21776 & 0.59786 \\
\hline & & & PR & 0.026292 & 0.009506 & 0.02904 & 0.020641 & 0.003845 \\
\hline & 300 & SELF & $\mathrm{BE}$ & 1.85822 & 2.57683 & 3.34688 & 0.21291 & 0.59656 \\
\hline & & & PR & 0.063635 & 0.042874 & 0.228372 & 0.000642 & 0.000918 \\
\hline & & PLF & $\mathrm{BE}$ & 1.87119 & 2.58445 & 3.39781 & 0.21401 & 0.59728 \\
\hline & & & PR & 0.033540 & 0.016527 & 0.067136 & 0.003005 & 0.001538 \\
\hline & & DLF & $\mathrm{BE}$ & 1.88059 & 2.60682 & 3.42132 & 0.21608 & 0.59795 \\
\hline & & & PR & 0.017818 & 0.006393 & 0.019735 & 0.013989 & 0.002580 \\
\hline
\end{tabular}


Table II: Bayes estimates (BEs) and posterior risks (PRs) of 3-component mixture of Gumbel type-II distributions using the JP under SELF, PLF and DLF with $\beta_{1}=2, \beta_{2}=3, \beta_{3}=4, p_{1}=0.20, p_{2}=0.60, t=15,20$

\begin{tabular}{|c|c|c|c|c|c|c|c|c|}
\hline \multirow[t]{2}{*}{$\mathrm{t}$} & \multirow[t]{2}{*}{$\mathrm{n}$} & \multirow{2}{*}{\multicolumn{2}{|c|}{ Loss functions }} & \multicolumn{5}{|c|}{$\mathrm{JP}$} \\
\hline & & & & $\hat{\beta}_{1}$ & $\hat{\beta}_{2}$ & $\hat{\beta}_{3}$ & $\hat{p}_{1}$ & $\hat{p}_{2}$ \\
\hline \multirow[t]{24}{*}{15} & \multirow[t]{6}{*}{50} & \multirow[t]{2}{*}{ SELF } & $\mathrm{BE}$ & 1.91359 & 2.45155 & 3.2509 & 0.22435 & 0.58195 \\
\hline & & & PR & 0.473989 & 0.252987 & 1.60348 & 0.003897 & 0.005413 \\
\hline & & \multirow[t]{2}{*}{ PLF } & $\mathrm{BE}$ & 1.97287 & 2.51875 & 3.52761 & 0.23276 & 0.58566 \\
\hline & & & PR & 0.212444 & 0.10088 & 0.44154 & 0.01714 & 0.00935 \\
\hline & & \multirow[t]{2}{*}{ DLF } & $\mathrm{BE}$ & 2.12995 & 2.56425 & 3.67571 & 0.24057 & 0.59092 \\
\hline & & & PR & 0.104298 & 0.0393711 & 0.119158 & 0.0731143 & 0.0158882 \\
\hline & \multirow[t]{6}{*}{100} & \multirow[t]{2}{*}{ SELF } & $\mathrm{BE}$ & 1.79531 & 2.43366 & 3.14736 & 0.22006 & 0.58906 \\
\hline & & & PR & 0.194814 & 0.122887 & 0.687328 & 0.002009 & 0.002815 \\
\hline & & \multirow[t]{2}{*}{ PLF } & $\mathrm{BE}$ & 1.84124 & 2.48089 & 3.21993 & 0.224475 & 0.59115 \\
\hline & & & PR & 0.102013 & 0.050157 & 0.204803 & 0.009063 & 0.004791 \\
\hline & & \multirow[t]{2}{*}{ DLF } & $\mathrm{BE}$ & 1.86886 & 2.50321 & 3.38474 & 0.22953 & 0.59462 \\
\hline & & & PR & 0.054494 & 0.020077 & 0.062901 & 0.040128 & 0.008080 \\
\hline & \multirow[t]{6}{*}{200} & \multirow[t]{2}{*}{ SELF } & $\mathrm{BE}$ & 1.75336 & 2.4217 & 3.08974 & 0.21828 & 0.59340 \\
\hline & & & PR & 0.090090 & 0.060389 & 0.321638 & 0.001023 & 0.001437 \\
\hline & & \multirow[t]{2}{*}{ PLF } & $\mathrm{BE}$ & 1.7756 & 2.43406 & 3.13438 & 0.22007 & 0.59628 \\
\hline & & & PR & 0.050067 & 0.024679 & 0.10183 & 0.004675 & 0.002416 \\
\hline & & \multirow[t]{2}{*}{ DLF } & $\mathrm{BE}$ & 1.79672 & 2.4459 & 3.18386 & 0.22295 & 0.59565 \\
\hline & & & PR & 0.027906 & 0.010143 & 0.031971 & 0.021133 & 0.004085 \\
\hline & \multirow[t]{6}{*}{300} & \multirow[t]{2}{*}{ SELF } & $\mathrm{BE}$ & 1.73697 & 2.43213 & 3.06366 & 0.21697 & 0.59573 \\
\hline & & & PR & 0.058619 & 0.040471 & 0.209437 & 0.000685 & 0.000965 \\
\hline & & \multirow[t]{2}{*}{ PLF } & $\mathrm{BE}$ & 1.75734 & 2.42595 & 3.0954 & 0.21841 & 0.59728 \\
\hline & & & PR & 0.033207 & 0.016422 & 0.067263 & 0.003148 & 0.001617 \\
\hline & & DLF & $\mathrm{BE}$ & 1.77108 & 2.44365 & 3.12952 & 0.2213 & 0.59661 \\
\hline & & & PR & 0.018707 & 0.006779 & 0.021598 & 0.014293 & 0.002727 \\
\hline 20 & 50 & SELF & $\mathrm{BE}$ & 1.98608 & 2.62234 & 3.58365 & 0.22047 & 0.58209 \\
\hline & & & PR & 0.488933 & 0.276524 & 1.7856 & 0.003668 & 0.005166 \\
\hline & & PLF & $\mathrm{BE}$ & 2.05258 & 2.67762 & 3.6995 & 0.22897 & 0.58573 \\
\hline & & & PR & 0.210662 & 0.101463 & 0.42616 & 0.016341 & 0.008885 \\
\hline & & DLF & $\mathrm{BE}$ & 2.16879 & 2.74603 & 3.93224 & 0.23758 & 0.59052 \\
\hline & & & PR & 0.100202 & 0.037637 & 0.112275 & 0.070830 & 0.015206 \\
\hline & 100 & SELF & $\mathrm{BE}$ & 1.85242 & 2.58929 & 3.40073 & 0.21547 & 0.59044 \\
\hline & & & PR & 0.200264 & 0.132206 & 0.748278 & 0.001886 & 0.002686 \\
\hline & & PLF & $\mathrm{BE}$ & 1.92981 & 2.60099 & 3.52903 & 0.22024 & 0.59309 \\
\hline & & & PR & 0.102819 & 0.049756 & 0.209779 & 0.008652 & 0.004536 \\
\hline & & DLF & $\mathrm{BE}$ & 1.94744 & 2.65098 & 3.63798 & 0.22414 & 0.59434 \\
\hline & & & PR & 0.052889 & 0.019140 & 0.058144 & 0.039334 & 0.007710 \\
\hline & 200 & SELF & $\mathrm{BE}$ & 1.82224 & 2.56221 & 3.31138 & 0.21249 & 0.59504 \\
\hline & & & PR & 0.094558 & 0.064152 & 0.342771 & 0.000954 & 0.001367 \\
\hline & & PLF & $\mathrm{BE}$ & 1.84911 & 2.58254 & 3.38609 & 0.21616 & 0.59609 \\
\hline & & & PR & 0.050298 & 0.024948 & 0.102469 & 0.004472 & 0.002305 \\
\hline & & DLF & $\mathrm{BE}$ & 1.88254 & 2.5993 & 3.41256 & 0.21774 & 0.59728 \\
\hline & & & PR & 0.027018 & 0.009611 & 0.029842 & 0.020653 & 0.003856 \\
\hline & 300 & SELF & $\mathrm{BE}$ & 1.82188 & 2.56824 & 3.30343 & 0.21249 & 0.59504 \\
\hline & & & PR & 0.094558 & 0.064152 & 0.226589 & 0.000640 & 0.000917 \\
\hline & & PLF & $\mathrm{BE}$ & 1.83324 & 2.58679 & 3.30813 & 0.21407 & 0.59740 \\
\hline & & & PR & 0.033455 & 0.016643 & 0.066773 & 0.003005 & 0.001538 \\
\hline & & DLF & $\mathrm{BE}$ & 1.85456 & 2.58981 & 3.37147 & 0.21540 & 0.59811 \\
\hline & & & PR & 0.018178 & 0.006425 & 0.020056 & 0.014029 & 0.002576 \\
\hline
\end{tabular}


Table III: Bayes estimates (BEs) and posterior risks (PRs) of 3-component mixture of Gumbel type-II distributions using the UP under SELF, PLF and DLF with $\beta_{1}=3, \beta_{2}=3, \beta_{3}=3, p_{1}=0.40, p_{2}=0.40, t=15,20$

\begin{tabular}{|c|c|c|c|c|c|c|c|c|}
\hline \multirow[t]{2}{*}{$\mathrm{t}$} & \multirow[t]{2}{*}{$\mathrm{n}$} & \multirow{2}{*}{\multicolumn{2}{|c|}{ Loss functions }} & \multicolumn{5}{|c|}{ UP } \\
\hline & & & & $\hat{\beta}_{1}$ & $\hat{\beta}_{2}$ & $\hat{\beta}_{3}$ & $\hat{p}_{1}$ & $\hat{p}_{2}$ \\
\hline \multirow[t]{24}{*}{15} & \multirow[t]{6}{*}{50} & \multirow[t]{2}{*}{ SELF } & $\mathrm{BE}$ & 2.62793 & 2.64591 & 2.95677 & 0.39521 & 0.39604 \\
\hline & & & PR & 0.421742 & 0.425329 & 1.07593 & 0.005321 & 0.005323 \\
\hline & & \multirow[t]{2}{*}{ PLF } & $\mathrm{BE}$ & 2.70489 & 2.75525 & 3.08741 & 0.40154 & 0.40333 \\
\hline & & & PR & 0.152277 & 0.154277 & 0.321992 & 0.013436 & 0.013394 \\
\hline & & \multirow[t]{2}{*}{ DLF } & $\mathrm{BE}$ & 2.81627 & 2.81706 & 3.24022 & 0.40897 & 0.40965 \\
\hline & & & PR & 0.055552 & 0.055434 & 0.101097 & 0.033555 & 0.033465 \\
\hline & \multirow[t]{6}{*}{100} & \multirow[t]{2}{*}{ SELF } & $\mathrm{BE}$ & 2.5562 & 2.54079 & 2.63976 & 0.39839 & 0.39763 \\
\hline & & & PR & 0.199723 & 0.197194 & 0.427289 & 0.002797 & 0.002795 \\
\hline & & \multirow[t]{2}{*}{ PLF } & $\mathrm{BE}$ & 2.57602 & 2.56665 & 2.74725 & 0.40174 & 0.40104 \\
\hline & & & PR & 0.07546 & 0.075397 & 0.154999 & 0.007018 & 0.007026 \\
\hline & & \multirow[t]{2}{*}{ DLF } & $\mathrm{BE}$ & 2.63711 & 2.63325 & 2.82425 & 0.40435 & 0.40506 \\
\hline & & & PR & 0.029048 & 0.028992 & 0.055161 & 0.017479 & 0.017425 \\
\hline & \multirow[t]{6}{*}{200} & \multirow[t]{2}{*}{ SELF } & $\mathrm{BE}$ & 2.48608 & 2.47612 & 2.54494 & 0.39995 & 0.39856 \\
\hline & & & PR & 0.094380 & 0.093854 & 0.199052 & 0.001434 & 0.001432 \\
\hline & & \multirow[t]{2}{*}{ PLF } & $\mathrm{BE}$ & 2.47993 & 2.49275 & 2.5724 & 0.40106 & 0.40089 \\
\hline & & & PR & 0.037096 & 0.037296 & 0.075674 & 0.003581 & 0.003582 \\
\hline & & \multirow[t]{2}{*}{ DLF } & $\mathrm{BE}$ & 2.51629 & 2.5166 & 2.58785 & 0.40217 & 0.40237 \\
\hline & & & $\mathrm{PR}$ & 0.014923 & 0.014913 & 0.029039 & 0.008956 & 0.008947 \\
\hline & \multirow[t]{6}{*}{300} & \multirow[t]{2}{*}{ SELF } & $\mathrm{BE}$ & 2.45501 & 2.44369 & 2.49606 & 0.39848 & 0.39954 \\
\hline & & & PR & 0.061591 & 0.060864 & 0.126877 & 0.000961 & 0.000962 \\
\hline & & \multirow[t]{2}{*}{ PLF } & $\mathrm{BE}$ & 2.47862 & 2.47159 & 2.53897 & 0.40093 & 0.40174 \\
\hline & & & PR & 0.010058 & 0.010038 & 0.019690 & 0.006035 & 0.006015 \\
\hline & & DLF & $\mathrm{BE}$ & 2.47185 & 2.46913 & 2.5463 & 0.40141 & 0.40250 \\
\hline & & & PR & 0.010050 & 0.010024 & 0.019822 & 0.006025 & 0.005998 \\
\hline 20 & 50 & SELF & $\mathrm{BE}$ & 2.82852 & 2.8412 & 3.12293 & 0.39401 & 0.39794 \\
\hline & & & $\mathrm{PR}$ & 0.465625 & 0.466401 & 1.14887 & 0.005075 & 0.005093 \\
\hline & & PLF & $\mathrm{BE}$ & 2.90408 & 2.88555 & 3.26087 & 0.40227 & 0.40279 \\
\hline & & & PR & 0.154803 & 0.153578 & 0.32237 & 0.012791 & 0.012777 \\
\hline & & DLF & $\mathrm{BE}$ & 2.99341 & 2.93687 & 3.43063 & 0.40983 & 0.40748 \\
\hline & & & PR & 0.052295 & 0.052619 & 0.095686 & 0.031563 & 0.031883 \\
\hline & 100 & SELF & $\mathrm{BE}$ & 2.70475 & 2.70685 & 2.7989 & 0.39821 & 0.39638 \\
\hline & & & PR & 0.21215 & 0.213714 & 0.453306 & 0.002661 & 0.002656 \\
\hline & & PLF & $\mathrm{BE}$ & 2.72495 & 2.71639 & 2.90887 & 0.40162 & 0.40074 \\
\hline & & & $\mathrm{PR}$ & 0.075495 & 0.075436 & 0.154702 & 0.006650 & 0.006660 \\
\hline & & DLF & $\mathrm{BE}$ & 2.76686 & 2.76649 & 2.99525 & 0.40457 & 0.40399 \\
\hline & & & PR & 0.027602 & 0.027633 & 0.052424 & 0.016619 & 0.016649 \\
\hline & 200 & SELF & $\mathrm{BE}$ & 2.61974 & 2.61182 & 2.6998 & 0.39916 & 0.39873 \\
\hline & & & PR & 0.099579 & 0.099068 & 0.211307 & 0.001361 & 0.001361 \\
\hline & & PLF & $\mathrm{BE}$ & 2.63904 & 2.63855 & 2.73 & 0.40132 & 0.39985 \\
\hline & & & PR & 0.037443 & 0.037568 & 0.075892 & 0.003404 & 0.003412 \\
\hline & & DLF & $\mathrm{BE}$ & 2.66335 & 2.66555 & 2.76496 & 0.401336 & 0.40263 \\
\hline & & & PR & 0.014202 & 0.014156 & 0.027536 & 0.008544 & 0.008498 \\
\hline & 300 & SELF & $\mathrm{BE}$ & 2.59774 & 2.59539 & 2.63784 & 0.39879 & 0.39944 \\
\hline & & & PR & 0.065525 & 0.065301 & 0.134688 & 0.000916 & 0.000916 \\
\hline & & PLF & $\mathrm{BE}$ & 2.61575 & 2.59533 & 2.6644 & 0.40040 & 0.40008 \\
\hline & & & PR & 0.024985 & 0.024815 & 0.050119 & 0.002290 & 0.002292 \\
\hline & & DLF & $\mathrm{BE}$ & 2.62345 & 2.62279 & 2.69464 & 0.40226 & 0.40097 \\
\hline & & & PR & 0.009508 & 0.009539 & 0.018746 & 0.005699 & 0.005730 \\
\hline
\end{tabular}


Table IV: Bayes estimates (BEs) and posterior risks (PRs) of 3-component mixture of Gumbel type-II distributions using the JP under SELF, PLF and DLF with $\beta_{1}=3, \beta_{2}=3, \beta_{3}=3, p_{1}=0.40, p_{2}=0.40, t=15,20$

\begin{tabular}{|c|c|c|c|c|c|c|c|c|}
\hline \multirow[t]{2}{*}{$\mathrm{t}$} & \multirow[t]{2}{*}{$\mathrm{n}$} & \multirow{2}{*}{\multicolumn{2}{|c|}{ Loss functions }} & \multicolumn{5}{|c|}{ JP } \\
\hline & & & & $\hat{\beta}_{1}$ & $\hat{\beta}_{2}$ & $\hat{\beta}_{3}$ & $\hat{p}_{1}$ & $\hat{p}_{2}$ \\
\hline \multirow[t]{24}{*}{15} & \multirow[t]{6}{*}{50} & \multirow[t]{2}{*}{ SELF } & $\mathrm{BE}$ & 2.50579 & 2.51536 & 2.64102 & 0.39644 & 0.39490 \\
\hline & & & PR & 0.402872 & 0.406845 & 0.957829 & 0.005300 & 0.005292 \\
\hline & & \multirow[t]{2}{*}{ PLF } & $\mathrm{BE}$ & 2.57686 & 2.63748 & 2.77313 & 0.40212 & 0.40141 \\
\hline & & & PR & 0.152588 & 0.156486 & 0.318867 & 0.013379 & 0.013393 \\
\hline & & \multirow[t]{2}{*}{ DLF } & $\mathrm{BE}$ & 2.66136 & 2.67441 & 2.93516 & 0.40854 & 0.40841 \\
\hline & & & PR & 0.058533 & 0.058548 & 0.11116 & 0.033449 & 0.033467 \\
\hline & \multirow[t]{6}{*}{100} & \multirow[t]{2}{*}{ SELF } & $\mathrm{BE}$ & 2.46833 & 2.45947 & 2.48678 & 0.39648 & 0.39748 \\
\hline & & & PR & 0.191831 & 0.190109 & 0.396216 & 0.002786 & 0.002789 \\
\hline & & \multirow[t]{2}{*}{ PLF } & $\mathrm{BE}$ & 2.48161 & 2.50522 & 2.60458 & 0.40145 & 0.40131 \\
\hline & & & PR & 0.074904 & 0.075625 & 0.155267 & 0.007013 & 0.007015 \\
\hline & & \multirow[t]{2}{*}{ DLF } & $\mathrm{BE}$ & 2.53792 & 2.53114 & 2.67258 & 0.40506 & 0.40403 \\
\hline & & & PR & 0.029799 & 0.029891 & 0.058240 & 0.017395 & 0.017480 \\
\hline & \multirow[t]{6}{*}{200} & \multirow[t]{2}{*}{ SELF } & $\mathrm{BE}$ & 2.42514 & 2.44289 & 2.4845 & 0.39850 & 0.39877 \\
\hline & & & PR & 0.091510 & 0.092750 & 0.194863 & 0.001431 & 0.001431 \\
\hline & & \multirow[t]{2}{*}{ PLF } & $\mathrm{BE}$ & 2.45645 & 2.45217 & 2.48111 & 0.39999 & 0.40112 \\
\hline & & & PR & 0.0374 & 0.037233 & 0.074844 & 0.003587 & 0.003580 \\
\hline & & \multirow[t]{2}{*}{ DLF } & $\mathrm{BE}$ & 2.47872 & 2.46598 & 2.52285 & 0.40259 & 0.40242 \\
\hline & & & PR & 0.015137 & 0.015142 & 0.029992 & 0.008945 & 0.008949 \\
\hline & \multirow[t]{6}{*}{300} & \multirow[t]{2}{*}{ SELF } & $\mathrm{BE}$ & 2.42342 & 2.41642 & 2.45373 & 0.39863 & 0.39971 \\
\hline & & & PR & 0.060662 & 0.060112 & 0.125576 & 0.000962 & 0.000962 \\
\hline & & \multirow[t]{2}{*}{ PLF } & $\mathrm{BE}$ & 2.44252 & 2.43282 & 2.47083 & 0.40024 & 0.40036 \\
\hline & & & PR & 0.024837 & 0.024733 & 0.049966 & 0.002409 & 0.002408 \\
\hline & & DLF & $\mathrm{BE}$ & 2.46121 & 2.45183 & 2.49706 & 0.40183 & 0.40168 \\
\hline & & & PR & 0.010131 & 0.010135 & 0.020166 & 0.006009 & 0.006013 \\
\hline 20 & 50 & SELF & $\mathrm{BE}$ & 2.68043 & 2.6468 & 2.80766 & 0.39553 & 0.39569 \\
\hline & & & PR & 0.44025 & 0.430049 & 1.03467 & 0.005073 & 0.005075 \\
\hline & & PLF & $\mathrm{BE}$ & 2.74963 & 2.7575 & 2.9669 & 0.40222 & 0.40176 \\
\hline & & & PR & 0.154661 & 0.155583 & 0.324787 & 0.012783 & 0.012792 \\
\hline & & DLF & $\mathrm{BE}$ & 2.81269 & 2.80334 & 3.14895 & 0.40840 & 0.40935 \\
\hline & & & PR & 0.055680 & 0.055555 & 0.10659 & 0.031909 & 0.031783 \\
\hline & 100 & SELF & $\mathrm{BE}$ & 2.5937 & 2.59228 & 2.65418 & 0.39748 & 0.39741 \\
\hline & & & PR & 0.200978 & 0.201306 & 0.429992 & 0.002662 & 0.002662 \\
\hline & & PLF & $\mathrm{BE}$ & 2.66345 & 2.637 & 2.74595 & 0.40147 & 0.40063 \\
\hline & & & PR & 0.076000 & 0.075421 & 0.154451 & 0.003403 & 0.003408 \\
\hline & & DLF & $\mathrm{BE}$ & 2.71343 & 2.68486 & 2.81504 & 0.40482 & 0.40401 \\
\hline & & & PR & 0.028407 & 0.028475 & 0.055509 & 0.016624 & 0.016686 \\
\hline & 200 & SELF & $\mathrm{BE}$ & 2.57921 & 2.59528 & 2.611 & 0.39828 & 0.39922 \\
\hline & & & PR & 0.098256 & 0.099295 & 0.203591 & 0.001362 & 0.001363 \\
\hline & & PLF & $\mathrm{BE}$ & 2.62219 & 2.58364 & 2.65262 & 0.40010 & 0.40025 \\
\hline & & & PR & 0.037722 & 0.037246 & 0.075879 & 0.003403 & 0.003408 \\
\hline & & DLF & $\mathrm{BE}$ & 2.61636 & 2.61068 & 2.70208 & 0.40239 & 0.40180 \\
\hline & & & PR & 0.014380 & 0.014401 & 0.028380 & 0.008514 & 0.008534 \\
\hline & 300 & SELF & $\mathrm{BE}$ & 2.57043 & 2.57452 & 2.59734 & 0.39887 & 0.39941 \\
\hline & & & PR & 0.06469 & 0.064765 & 0.132917 & 0.000914 & 0.000915 \\
\hline & & PLF & $\mathrm{BE}$ & 2.57775 & 2.58242 & 2.61386 & 0.40098 & 0.40051 \\
\hline & & & PR & 0.024827 & 0.024906 & 0.050360 & 0.002289 & 0.002290 \\
\hline & & DLF & $\mathrm{BE}$ & 2.60664 & 2.6071 & 2.65028 & 0.40158 & 0.40194 \\
\hline & & & PR & 0.009625 & 0.009616 & 0.019149 & 0.005721 & 0.005713 \\
\hline
\end{tabular}


Table V: Bayes estimates (BEs) and posterior risks (PRs) of 3-component mixture of Gumbel type-II distributions using the GP under SELF, PLF and DLF with $\beta_{1}=2, \beta_{2}=3, \beta_{3}=4, a_{1}=1.7388, a_{2}=0.4722, a_{3}=0.3528, b_{1}=1.0779, b_{2}=0.1469$, $b_{3}=0.0737, a=0.3695, b=1.4360, c=8.8749, p_{1}=0.20, p_{2}=0.60, t=15,20$.

\begin{tabular}{|c|c|c|c|c|c|c|c|c|}
\hline \multirow[t]{2}{*}{$\mathrm{t}$} & \multirow[t]{2}{*}{$\mathrm{n}$} & \multirow{2}{*}{\multicolumn{2}{|c|}{ Loss functions }} & \multicolumn{5}{|c|}{ GP } \\
\hline & & & & $\hat{\beta}_{1}$ & $\hat{\beta}_{2}$ & $\hat{\beta}_{3}$ & $\hat{p}_{1}$ & $\hat{p}_{2}$ \\
\hline \multirow[t]{24}{*}{15} & \multirow[t]{6}{*}{50} & \multirow[t]{2}{*}{ SELF } & $\mathrm{BE}$ & 1.56683 & 2.40591 & 3.07652 & 0.23817 & 0.57133 \\
\hline & & & PR & 0.241388 & 0.207639 & 0.711495 & 0.003932 & 0.005267 \\
\hline & & \multirow[t]{2}{*}{ PLF } & $\mathrm{BE}$ & 1.66061 & 2.44352 & 3.19152 & 0.24824 & 0.57807 \\
\hline & & & PR & 0.145569 & 0.083929 & 0.226379 & 0.016248 & 0.009192 \\
\hline & & \multirow[t]{2}{*}{ DLF } & $\mathrm{BE}$ & 1.72694 & 2.4871 & 3.28841 & 0.25575 & 0.58198 \\
\hline & & & PR & 0.085907 & 0.034032 & 0.069234 & 0.065194 & 0.015959 \\
\hline & \multirow[t]{6}{*}{100} & \multirow[t]{2}{*}{ SELF } & $\mathrm{BE}$ & 1.65448 & 2.40522 & 3.07746 & 0.22797 & 0.585222 \\
\hline & & & PR & 0.146583 & 0.111094 & 0.46131 & 0.002038 & 0.002790 \\
\hline & & \multirow[t]{2}{*}{ PLF } & $\mathrm{BE}$ & 1.68095 & 2.43261 & 3.14103 & 0.23219 & 0.58733 \\
\hline & & & PR & 0.083698 & 0.045390 & 0.144118 & 0.008823 & 0.004750 \\
\hline & & \multirow[t]{2}{*}{ DLF } & $\mathrm{BE}$ & 1.73631 & 2.44626 & 3.19767 & 0.23654 & 0.59015 \\
\hline & & & PR & 0.049253 & 0.018571 & 0.045425 & 0.037906 & 0.008087 \\
\hline & \multirow[t]{6}{*}{200} & \multirow[t]{2}{*}{ SELF } & $\mathrm{BE}$ & 1.67789 & 2.40478 & 3.0596 & 0.22234 & 0.59080 \\
\hline & & & PR & 0.077589 & 0.057147 & 0.260907 & 0.001027 & 0.001426 \\
\hline & & \multirow[t]{2}{*}{ PLF } & $\mathrm{BE}$ & 1.70871 & 2.40322 & 3.10043 & 0.22439 & 0.59315 \\
\hline & & & PR & 0.045493 & 0.023430 & 0.084019 & 0.004606 & 0.002412 \\
\hline & & \multirow[t]{2}{*}{ DLF } & $\mathrm{BE}$ & 1.78005 & 2.60856 & 3.46066 & 0.24920 & 0.58346 \\
\hline & & & PR & 0.083875 & 0.032581 & 0.066443 & 0.064252 & 0.015133 \\
\hline & \multirow[t]{6}{*}{300} & \multirow[t]{2}{*}{ SELF } & $\mathrm{BE}$ & 1.688 & 2.40726 & 3.06311 & 0.22019 & 0.59429 \\
\hline & & & PR & 0.053211 & 0.038604 & 0.184811 & 0.000689 & 0.000961 \\
\hline & & \multirow[t]{2}{*}{ PLF } & $\mathrm{BE}$ & 1.70816 & 2.42443 & 3.09497 & 0.22206 & 0.59540 \\
\hline & & & PR & 0.031020 & 0.016004 & 0.059690 & 0.003119 & 0.001617 \\
\hline & & DLF & $\mathrm{BE}$ & 1.73084 & 2.42641 & 3.09362 & 0.22294 & 0.59590 \\
\hline & & & PR & 0.018117 & 0.006588 & 0.019093 & 0.014073 & 0.002718 \\
\hline 20 & 50 & SELF & $\mathrm{BE}$ & 1.63206 & 2.52061 & 3.2088 & 0.23250 & 0.57498 \\
\hline & & & PR & 0.2555 & 0.217936 & 0.749546 & 0.003695 & 0.005036 \\
\hline & & PLF & $\mathrm{BE}$ & 1.69767 & 2.54503 & 3.34103 & 0.24197 & 0.57906 \\
\hline & & & PR & 0.14462 & 0.083487 & 0.226435 & 0.015594 & 0.008744 \\
\hline & & DLF & $\mathrm{BE}$ & 1.78005 & 2.60856 & 3.46066 & 0.24920 & 0.58346 \\
\hline & & & PR & 0.083875 & 0.032581 & 0.066443 & 0.064252 & 0.015133 \\
\hline & 100 & SELF & $\mathrm{BE}$ & 1.71018 & 2.5279 & 3.26042 & 0.22321 & 0.58629 \\
\hline & & & PR & 0.150658 & 0.116468 & 0.489156 & 0.001901 & 0.002647 \\
\hline & & PLF & $\mathrm{BE}$ & 1.74817 & 2.55661 & 3.29384 & 0.22741 & 0.58849 \\
\hline & & & PR & 0.084285 & 0.045499 & 0.143777 & 0.008449 & 0.004518 \\
\hline & & DLF & $\mathrm{BE}$ & 1.7895 & 2.57899 & 3.35566 & 0.23192 & 0.59098 \\
\hline & & & PR & 0.047566 & 0.017695 & 0.043204 & 0.036876 & 0.007668 \\
\hline & 200 & SELF & $\mathrm{BE}$ & 1.75244 & 2.52875 & 3.25501 & 0.21805 & 0.59295 \\
\hline & & & PR & 0.081961 & 0.060124 & 0.280411 & 0.000964 & 0.001359 \\
\hline & & PLF & $\mathrm{BE}$ & 1.77711 & 2.55312 & 3.28737 & 0.21942 & 0.59456 \\
\hline & & & PR & 0.045908 & 0.02368 & 0.084041 & 0.004408 & 0.002290 \\
\hline & & DLF & $\mathrm{BE}$ & 1.80335 & 2.57442 & 3.34172 & 0.22243 & 0.59505 \\
\hline & & & PR & 0.025536 & 0.009256 & 0.025407 & 0.019926 & 0.003861 \\
\hline & 300 & SELF & $\mathrm{BE}$ & 1.7727 & 2.54839 & 3.26254 & 0.21506 & 0.59560 \\
\hline & & & PR & 0.056899 & 0.041106 & 0.196652 & 0.000643 & 0.000914 \\
\hline & & PLF & $\mathrm{BE}$ & 1.77372 & 2.56268 & 3.2735 & 0.21699 & 0.59544 \\
\hline & & & PR & 0.031184 & 0.016086 & 0.058896 & 0.002978 & 0.001537 \\
\hline & & DLF & $\mathrm{BE}$ & 1.7855 & 2.57433 & 3.32566 & 0.21837 & 0.59626 \\
\hline & & & PR & 0.017496 & 0.006262 & 0.017886 & 0.013696 & 0.002579 \\
\hline
\end{tabular}


Table VI: Bayes estimates (BEs) and posterior risks (PRs) of 3-component mixture of Gumbel type-II distributions using the GP under SELF, PLF and DLF with $\beta_{1}=3, \beta_{2}=3, \beta_{3}=3, a_{1}=1.7388, a_{2}=0.4722, a_{3}=0.3528, b_{1}=1.0779, b_{2}=0.1469$, $b_{3}=0.0737, a=0.3695, b=1.4360, c=8.8749, p_{1}=0.40, p_{2}=0.40, \mathrm{t}=15,20$

\begin{tabular}{|c|c|c|c|c|c|c|c|c|}
\hline \multirow[t]{2}{*}{$\mathrm{T}$} & \multirow[t]{2}{*}{$\mathrm{n}$} & \multicolumn{2}{|c|}{ Loss functions } & \multicolumn{5}{|c|}{ GP } \\
\hline & & & & $\hat{\beta}_{1}$ & $\hat{\beta}_{2}$ & $\hat{\beta}_{3}$ & $\hat{p}_{1}$ & $\hat{p}_{2}$ \\
\hline \multirow[t]{24}{*}{15} & \multirow[t]{6}{*}{50} & SELF & $\mathrm{BE}$ & 2.12678 & 2.38951 & 2.72082 & 0.40564 & 0.39293 \\
\hline & & & PR & 0.254593 & 0.289911 & 0.544987 & 0.005204 & 0.005144 \\
\hline & & PLF & $\mathrm{BE}$ & 2.18569 & 2.43408 & 2.82963 & 0.41106 & 0.40064 \\
\hline & & & PR & 0.115597 & 0.115898 & 0.191636 & 0.012760 & 0.012965 \\
\hline & & DLF & $\mathrm{BE}$ & 2.2665 & 2.48761 & 2.89171 & 0.41782 & 0.40747 \\
\hline & & & PR & 0.052405 & 0.047261 & 0.066818 & 0.031160 & 0.032519 \\
\hline & \multirow[t]{6}{*}{100} & SELF & $\mathrm{BE}$ & 2.25086 & 2.3792 & 2.6293 & 0.40268 & 0.39659 \\
\hline & & & PR & 0.149246 & 0.157632 & 0.319119 & 0.002756 & 0.002740 \\
\hline & & PLF & $\mathrm{BE}$ & 2.30602 & 2.40795 & 2.66264 & 0.40645 & 0.39950 \\
\hline & & & PR & 0.065449 & 0.064773 & 0.1162 & 0.006823 & 0.006899 \\
\hline & & DLF & $\mathrm{BE}$ & 2.32588 & 2.46535 & 2.71347 & 0.40958 & 0.40366 \\
\hline & & & PR & 0.028255 & 0.02671 & 0.043260 & 0.016847 & 0.017258 \\
\hline & \multirow[t]{6}{*}{200} & SELF & $\mathrm{BE}$ & 2.33068 & 2.40639 & 2.53466 & 0.40174 & 0.39823 \\
\hline & & & PR & 0.081609 & 0.084513 & 0.17035 & 0.001422 & 0.001417 \\
\hline & & PLF & $\mathrm{BE}$ & 2.35514 & 2.41406 & 2.5509 & 0.40375 & 0.39892 \\
\hline & & & PR & 0.03468 & 0.034658 & 0.064973 & 0.003533 & 0.003561 \\
\hline & & DLF & $\mathrm{BE}$ & 2.36777 & 2.43104 & 2.59392 & 0.40521 & 0.4023 \\
\hline & & & PR & 0.014684 & 0.014251 & 0.025453 & 0.008765 & 0.008868 \\
\hline & \multirow[t]{6}{*}{300} & SELF & $\mathrm{BE}$ & 2.35866 & 2.40545 & 2.48941 & 0.40083 & 0.39948 \\
\hline & & & PR & 0.056178 & 0.057160 & 0.114795 & 0.000958 & 0.000956 \\
\hline & & PLF & $\mathrm{BE}$ & 2.37855 & 2.41126 & 2.50286 & 0.40193 & 0.40030 \\
\hline & & & PR & 0.023701 & 0.023529 & 0.045154 & 0.002388 & 0.002394 \\
\hline & & DLF & $\mathrm{BE}$ & 2.37596 & 2.4273 & 2.54011 & 0.40338 & 0.40113 \\
\hline & & & PR & 0.009925 & 0.009731 & 0.017937 & 0.005930 & 0.005984 \\
\hline \multirow[t]{24}{*}{20} & \multirow[t]{6}{*}{50} & SELF & $\mathrm{BE}$ & 2.23761 & 2.48796 & 2.78189 & 0.40413 & 0.39282 \\
\hline & & & PR & 0.26815 & 0.301329 & 0.548209 & 0.004953 & 0.004903 \\
\hline & & PLF & $\mathrm{BE}$ & 2.29827 & 2.55371 & 2.89405 & 0.41080 & 0.39812 \\
\hline & & & PR & 0.115619 & 0.117149 & 0.188654 & 0.012185 & 0.012436 \\
\hline & & DLF & $\mathrm{BE}$ & 2.3717 & 2.62563 & 3.00873 & 0.41742 & 0.40687 \\
\hline & & & PR & 0.049823 & 0.045242 & 0.064752 & 0.029644 & 0.030952 \\
\hline & \multirow[t]{6}{*}{100} & SELF & $\mathrm{BE}$ & 2.39365 & 2.52706 & 2.71051 & 0.40296 & 0.39629 \\
\hline & & & PR & 0.160617 & 0.16999 & 0.327067 & 0.002632 & 0.002616 \\
\hline & & PLF & $\mathrm{BE}$ & 2.42274 & 2.56881 & 2.78415 & 0.40611 & 0.39986 \\
\hline & & & PR & 0.065507 & 0.065964 & 0.117108 & 0.006516 & 0.006580 \\
\hline & & DLF & $\mathrm{BE}$ & 2.47136 & 2.59433 & 2.85391 & 0.40957 & 0.40241 \\
\hline & & & PR & 0.026780 & 0.025501 & 0.041446 & 0.015980 & 0.016465 \\
\hline & \multirow[t]{6}{*}{200} & SELF & $\mathrm{BE}$ & 2.46999 & 2.53253 & 2.64858 & 0.40142 & 0.39787 \\
\hline & & & PR & 0.087172 & 0.089220 & 0.17707 & 0.001353 & 0.001349 \\
\hline & & PLF & $\mathrm{BE}$ & 2.48995 & 2.55163 & 2.68829 & 0.40324 & 0.40031 \\
\hline & & & PR & 0.034869 & 0.034753 & 0.065770 & 0.003366 & 0.003381 \\
\hline & & DLF & $\mathrm{BE}$ & 2.50759 & 2.58732 & 2.71896 & 0.40571 & 0.40082 \\
\hline & & & PR & 0.013930 & 0.013613 & 0.024274 & 0.008318 & 0.008487 \\
\hline & \multirow[t]{6}{*}{300} & SELF & $\mathrm{BE}$ & 2.49846 & 2.53969 & 2.62927 & 0.40121 & 0.39876 \\
\hline & & & PR & 0.059744 & 0.060617 & 0.12178 & 0.000910 & 0.000908 \\
\hline & & PLF & $\mathrm{BE}$ & 2.51474 & 2.5473 & 2.64388 & 0.40220 & 0.39991 \\
\hline & & & PR & 0.023784 & 0.023656 & 0.045509 & 0.002271 & 0.002279 \\
\hline & & DLF & $\mathrm{BE}$ & 2.51968 & 2.56658 & 2.67343 & 0.40341 & 0.40121 \\
\hline & & & PR & 0.009441 & 0.009269 & 0.017166 & 0.005648 & 0.005698 \\
\hline
\end{tabular}


Table VII: Bayes estimates and posterior risks (in parentheses) under SELF using complete sampling

\begin{tabular}{|c|c|c|c|c|c|}
\hline \multirow{2}{*}{$\begin{array}{c}\Phi=\left(\hat{\beta}_{1}, \hat{\beta}_{2}, \hat{\beta}_{3}, \hat{p}_{1}, \hat{p}_{2}\right) \\
\mathrm{n}\end{array}$} & \multicolumn{5}{|c|}{$\Phi=(2,3,4,0.20,0.60) \mathrm{UP}$} \\
\hline & $\hat{\beta}_{1}$ & $\hat{\beta}_{2}$ & $\hat{\beta}_{3}$ & $\hat{p}_{1}$ & $\hat{p}_{2}$ \\
\hline 50 & $\begin{array}{c}2.48265 \\
(0.629920)\end{array}$ & $\begin{array}{c}3.21679 \\
(0.345467)\end{array}$ & $\begin{array}{c}4.88026 \\
(2.437100)\end{array}$ & $\begin{array}{c}0.20755 \\
(0.003046)\end{array}$ & $\begin{array}{c}0.58491 \\
(0.004496)\end{array}$ \\
\hline 100 & $\begin{array}{c}2.22325 \\
(0.248929)\end{array}$ & $\begin{array}{c}3.09560 \\
(0.159958)\end{array}$ & $\begin{array}{c}4.44845 \\
(0.996820)\end{array}$ & $\begin{array}{c}0.20388 \\
(0.001561)\end{array}$ & $\begin{array}{c}0.59223 \\
(0.002322)\end{array}$ \\
\hline 200 & $\begin{array}{c}2.10990 \\
(0.111342)\end{array}$ & $\begin{array}{c}3.05416 \\
(0.077774)\end{array}$ & $\begin{array}{c}4.22293 \\
(0.446620)\end{array}$ & $\begin{array}{c}0.20197 \\
(0.000790)\end{array}$ & $\begin{array}{c}0.59606 \\
(0.001180)\end{array}$ \\
\hline 300 & $\begin{array}{c}2.06432 \\
(0.070969)\end{array}$ & $\begin{array}{c}3.04423 \\
(0.051500)\end{array}$ & $\begin{array}{c}4.13686 \\
(0.285329)\end{array}$ & $\begin{array}{c}0.20132 \\
(0.005289)\end{array}$ & $\begin{array}{c}0.59736 \\
(0.000791)\end{array}$ \\
\hline$\Phi=\left(\hat{\beta}_{1}, \hat{\beta}_{2}, \hat{\beta}_{3}, \hat{p}_{1}, \hat{p}_{2}\right)$ & \multicolumn{5}{|c|}{$\Phi=(2,3,4,0.20,0.60) \mathrm{JP}$} \\
\hline $\mathrm{n}$ & $\hat{\beta}_{1}$ & $\hat{\beta}_{2}$ & $\hat{\beta}_{3}$ & $\hat{p}_{1}$ & $\hat{p}_{2}$ \\
\hline 50 & $\begin{array}{c}2.21951 \\
(0.550923)\end{array}$ & $\begin{array}{c}3.08711 \\
(0.328661)\end{array}$ & $\begin{array}{c}4.36265 \\
(0.550923)\end{array}$ & $\begin{array}{c}0.20755 \\
(0.003046)\end{array}$ & $\begin{array}{c}0.58491 \\
(0.004496)\end{array}$ \\
\hline 100 & $\begin{array}{c}2.10056 \\
(0.235037)\end{array}$ & $\begin{array}{c}3.06126 \\
(0.158854)\end{array}$ & $\begin{array}{c}4.17918 \\
(0.235037)\end{array}$ & $\begin{array}{c}0.20388 \\
(0.001561)\end{array}$ & $\begin{array}{c}0.59223 \\
(0.002322)\end{array}$ \\
\hline 200 & $\begin{array}{c}2.04042 \\
(0.106829)\end{array}$ & $\begin{array}{c}3.02665 \\
(0.076988)\end{array}$ & $\begin{array}{c}4.12896 \\
(0.106829)\end{array}$ & $\begin{array}{c}0.20197 \\
(0.000790)\end{array}$ & $\begin{array}{c}0.59606 \\
(0.001180)\end{array}$ \\
\hline 300 & $\begin{array}{c}2.20698 \\
(0.069618)\end{array}$ & $\begin{array}{c}3.01377 \\
(0.050753)\end{array}$ & $\begin{array}{c}4.08431 \\
(0.069618)\end{array}$ & $\begin{array}{c}0.20132 \\
(0.000529)\end{array}$ & $\begin{array}{c}0.59736 \\
(0.000791)\end{array}$ \\
\hline$\Phi=\left(\hat{\beta}_{1}, \hat{\beta}_{2}, \hat{\beta}_{3}, \hat{p}_{1}, \hat{p}_{2}\right)$ & \multicolumn{5}{|c|}{$\Phi=(2,3,4,0.20,0.60) \mathrm{GP}$} \\
\hline $\mathrm{n}$ & $\hat{\beta}_{1}$ & $\hat{\beta}_{2}$ & $\hat{\beta}_{3}$ & $\hat{p}_{1}$ & $\hat{p}_{2}$ \\
\hline 50 & $\begin{array}{c}2.04143 \\
(0.379034)\end{array}$ & $\begin{array}{c}3.10604 \\
(0.326937)\end{array}$ & $\begin{array}{c}4.42386 \\
(2.101710)\end{array}$ & $\begin{array}{c}0.17089 \\
(0.002297)\end{array}$ & $\begin{array}{c}0.51806 \\
(0.004048)\end{array}$ \\
\hline 100 & $\begin{array}{c}2.04769 \\
(0.200731)\end{array}$ & $\begin{array}{c}3.05268 \\
(0.156494)\end{array}$ & $\begin{array}{c}4.26802 \\
(0.943645)\end{array}$ & $\begin{array}{c}0.18404 \\
(0.001345)\end{array}$ & $\begin{array}{c}0.55508 \\
(0.002211)\end{array}$ \\
\hline 200 & $\begin{array}{c}2.00909 \\
(0.098997)\end{array}$ & $\begin{array}{c}3.02822 \\
(0.076810)\end{array}$ & $\begin{array}{c}4.12159 \\
(0.431607)\end{array}$ & $\begin{array}{c}0.19162 \\
(0.000732)\end{array}$ & $\begin{array}{c}0.57640 \\
(0.001153)\end{array}$ \\
\hline 300 & $\begin{array}{c}2.02312 \\
(0.067357)\end{array}$ & $\begin{array}{c}3.02583 \\
(0.051004)\end{array}$ & $\begin{array}{c}4.08484 \\
(0.281109)\end{array}$ & $\begin{array}{c}0.19431 \\
(0.000502)\end{array}$ & $\begin{array}{c}0.58400 \\
(0.000779)\end{array}$ \\
\hline
\end{tabular}

